\title{
Identifying conductivity in electrical impedance tomography with total variation regularization
}

\author{
Michael Hinze ${ }^{d}$ ] Barbara Kaltenbacher ${ }^{t} \llbracket \cdot$ Tran Nhan Tam Quyen ${ }^{a, \uparrow}$ \\ ${ }^{a}$ University of Hamburg, Bundesstraße 55, D-20146 Hamburg, Germany \\ ${ }^{b}$ Alpen-Adria-Universität Klagenfurt, Universitätsstraße 65-67, A-9020 Klagenfurt, Austria
}

\begin{abstract}
In this paper we investigate the problem of identifying the conductivity in electrical impedance tomography from one boundary measurement. A variational method with total variation regularization is here proposed to tackle this problem. We discretize the PDE as well as the conductivity with piecewise linear, continuous finite elements. We prove the stability and convergence of this technique. For the numerical solution we propose a projected Armijo algorithm. Finally, a numerical experiment is presented to illustrate our theoretical results.
\end{abstract}

Key words and phrases: Conductivity identification, electrical impedance tomography, total variation regularization, finite element method, Neumann problem, Dirichlet problem, ill-posed problems.

AMS Subject Classifications: 65N21; 65N12; 35J25; 35R30

\section{Introduction}

Let $\Omega$ be an open bounded connected domain in $\mathbb{R}^{d}, d \in\{2,3\}$ with polygonal boundary $\partial \Omega$ and $f \in$ $H^{1}(\Omega)^{*}:=H^{-1}(\Omega)$ be given. We consider the following elliptic boundary value problem

$$
\begin{aligned}
-\nabla \cdot(q \nabla \Phi) & =f \text { in } \Omega, \\
q \nabla \Phi \cdot \vec{n} & =j^{\dagger} \text { on } \partial \Omega \text { and } \\
\Phi & =g^{\dagger} \text { on } \partial \Omega,
\end{aligned}
$$

where $\vec{n}$ is the unit outward normal on $\partial \Omega$.

The system 1.1 -1.3$)$ is overdetermined, i.e. if the Neumann and Dirichlet boundary conditions $j^{\dagger} \in$ $H^{-1 / 2}(\partial \Omega):=H^{1 / 2}(\partial \Omega)^{*}, g^{\dagger} \in H^{1 / 2}(\partial \Omega)$ and the conductivity

$$
q \in \mathcal{Q}:=\left\{q \in L^{\infty}(\Omega) \mid \underline{q} \leq q(x) \leq \bar{q} \text { a.e. in } \Omega\right\}
$$

are given, then there may be no $\Phi$ satisfying this system. Here $\underline{q}$ and $\bar{q}$ are some given positive constants.

In this paper we assume that the system is consistent and our aim is to identify the conductivity $q^{\dagger} \in \mathcal{Q}$ and the electric potential $\Phi^{\dagger} \in H^{1}(\Omega)$ in the system $11.1-1.3$ from current and voltage i.e., Neumann and Dirichlet measurements at the boundary $\left(j_{\delta}, g_{\delta}\right) \in H^{-1 / 2}(\partial \Omega) \times H^{1 / 2}(\partial \Omega)$ of the exact $\left(j^{\dagger}, g^{\dagger}\right)$ satisfying

$$
\left\|j_{\delta}-j^{\dagger}\right\|_{H^{-1 / 2}(\partial \Omega)}+\left\|g_{\delta}-g^{\dagger}\right\|_{H^{1 / 2}(\partial \Omega)} \leq \delta \text { with } \delta>0 .
$$

Note that using the $H^{-1 / 2}(\partial \Omega) \times H^{1 / 2}(\partial \Omega)$ topology for the data is natural from the point of view of solution theory for elliptic PDEs but unrealistic with regard to practical measurements. We will comment in this issue in Remark 2.2 below.

For the purpose of conductivity identification - a problem which is very well known in literature and practice as electrical impedance tomography EIT, see below for some references — we simultaneously consider the Neumann problem

$$
-\nabla \cdot(q \nabla u)=f \text { in } \Omega \text { and } q \nabla u \cdot \vec{n}=j_{\delta} \text { on } \partial \Omega
$$

Email: michael.hinze@uni-hamburg.de, Barbara.Kaltenbacher@aau.at, quyen.tran@uni-hamburg.de

M. Hinze gratefully acknowledges support of the Lothar Collatz Center for Computing in Science at the University of Hamburg

B. Kaltenbacher gratefully acknowledges support of the Austrian Wissenschaftsfonds through grant FWF I2271 entitled "Solving inverse problems without forward operators"

T.N.T. Quyen gratefully acknowledges support of the Alexander von Humboldt-Foundation

${ }^{c}$ Author to whom any correspondence should be addressed 
and the Dirichlet problem

$$
-\nabla \cdot(q \nabla v)=f \text { in } \Omega \text { and } v=g_{\delta} \text { on } \partial \Omega
$$

and respectively denote by $\mathcal{N}_{q} j_{\delta}, \mathcal{D}_{q} g_{\delta}$ the unique weak solutions of the problems (1.5), 1.6), which depend nonlinearly on $q$, where $\mathcal{N}_{q} j_{\delta}$ is normalized with vanishing mean on the boundary. We adopt the variational approach of Kohn and Vogelius in [30, 31, 32 to the identification problem. In fact, for estimating the conductivity $q$ from the observation $\left(j_{\delta}, g_{\delta}\right)$ of the exact data $\left(j^{\dagger}, g^{\dagger}\right)$, we use the functional

$$
\mathcal{J}_{\delta}(q):=\int_{\Omega} q \nabla\left(\mathcal{N}_{q} j_{\delta}-\mathcal{D}_{q} g_{\delta}\right) \cdot \nabla\left(\mathcal{N}_{q} j_{\delta}-\mathcal{D}_{q} g_{\delta}\right) d x
$$

For simplicity of exposition we restrict ourselves to the case of just one Neumann-Dirichlet pair, while the approach described here can be easily extended to multiple measurements $\left(j_{\delta}^{i}, g_{\delta}^{i}\right)_{i=1, \ldots, I}$, see also Example 5.3 below. It is well-known that such a finite number of boundary data in general only allows to identify conductivities taking finitely many different values in the domain $\Omega$, see, e.g., [2].

Indeed, we are interested in estimating such piecewise constant conductivities and therefore use total variation regularization, i.e., we consider the minimization problem

$$
\min _{q \in \mathcal{Q}_{a d}} \mathcal{J}_{\delta}(q)+\rho \int_{\Omega}|\nabla q|,
$$

where $\mathcal{Q}_{a d}:=\mathcal{Q} \cap B V(\Omega)$ is the admissible set of the sought conductivities, $B V(\Omega)$ is the space of all functions with bounded total variation (see $\$ 2.1$ for its definition) and $\rho>0$ is the regularization parameter, and consider a minimizer $q_{\rho, \delta}$ of (1.7) as reconstruction.

For each $q \in \mathcal{Q}$ let $\mathcal{N}_{q}^{h} j_{\delta}$ and $\mathcal{D}_{q}^{h} g_{\delta}$ be corresponding approximations of $\mathcal{N}_{q} j_{\delta}$ and $\mathcal{D}_{q} g_{\delta}$ in the finite dimensional space $\mathcal{V}_{1}^{h}$ of piecewise linear, continuous finite elements and $q_{\rho, \delta}^{h}$ denote a minimizer of the discrete regularized problem corresponding to (1.7), i.e. of the following minimization problem

$$
\min _{q \in \mathcal{Q}_{a d}^{h}} \int_{\Omega} q \nabla\left(\mathcal{N}_{q}^{h} j_{\delta}-\mathcal{D}_{q}^{h} g_{\delta}\right) \cdot \nabla\left(\mathcal{N}_{q}^{h} j_{\delta}-\mathcal{D}_{q}^{h} g_{\delta}\right) d x+\rho \int_{\Omega} \sqrt{|\nabla q|^{2}+\epsilon^{h}}
$$

with $\mathcal{Q}_{a d}^{h}:=\mathcal{Q}_{a d} \cap \mathcal{V}_{1}^{h}$ and $\epsilon^{h}$ being a positive functional of the mesh size $h$ satisfying $\lim _{h \rightarrow 0} \epsilon^{h}=0$.

In Section 4 we will show the stability of approximations for fixed positive $\rho$. Furthermore as $h, \delta \rightarrow 0$ and with an appropriate a priori regularization parameter choice $\rho=\rho(h, \delta)$, there exists a subsequence of $\left(q_{\rho, \delta}^{h}\right)$ converging in the $L^{1}(\Omega)$-norm to a total variation-minimizing solution $q^{\dagger}$ defined by

$$
q^{\dagger} \in \arg \min _{\left\{q \in \mathcal{Q}_{a d} \mid \mathcal{N}_{q} j^{\dagger}=\mathcal{D}_{q} g^{\dagger}\right\}} \int_{\Omega}|\nabla q| .
$$

In particular, if $q^{\dagger}$ is uniquely defined, then this convergence holds for the whole sequence $\left(q_{\rho, \delta}^{h}\right)$. The corresponding state sequences $\left(\mathcal{N}_{q_{\rho, \delta}^{h}}^{h} j_{\delta}\right)$ and $\left(\mathcal{D}_{q_{\rho, \delta}^{h}}^{h} g_{\delta}\right)$ converge in the $H^{1}(\Omega)$-norm to $\Phi^{\dagger}=\Phi^{\dagger}\left(q^{\dagger}, j^{\dagger}, g^{\dagger}\right)$ solving the system (1.1)-(1.3). Finally, for the numerical solution of the discrete regularized problem (1.8), in Section 5 we employ a projected Armijo algorithm. Numerical results show the efficiency of the proposed method and illustrate our theoretical findings.

We conclude this introduction with a selection of references from the vast literature on EIT, which has evolved to a highly relevant imaging and diagnostics tool in industrial and medical applications and has attracted great attention of many scientists in the last few decades.

To this end, for any fixed $q \in \mathcal{Q}$ we define the Neumann-to-Dirichlet map $\Lambda_{q}: H^{-1 / 2}(\partial \Omega) \rightarrow H^{1 / 2}(\partial \Omega)$, by

$$
j \mapsto \Lambda_{q} j:=\gamma \mathcal{N}_{q} j,
$$

where $\gamma: H^{1}(\Omega) \rightarrow H^{1 / 2}(\partial \Omega)$ is the Dirichlet trace operator. Calderón in 1980 posed the question whether an unknown conductivity distribution inside a domain can be determined from an infinite number of boundary observations, i.e. from the Neumann-to-Dirichlet map $\Lambda_{q}$ :

$$
p, q \in \mathcal{Q} \subset L^{\infty}(\Omega) \text { with } \Lambda_{p}=\Lambda_{q} \quad \Rightarrow \quad p=q ?
$$


Calderón did not answer his question (1.9); however, in [15] he proved that the problem linearized at constant conductivities has a unique solution. In dimensions three and higher Sylvester and Uhlmann 41 proved the unique identifiability of a $C^{\infty}$-smooth conductivity. Päivärinta el al. [37] and Brown and Torres [12] established uniqueness in the inverse conductivity problem for $W^{3 / 2, p}$-smooth conductivities with $p=\infty$ and $p>2 d$, respectively. In the two dimensional setting, Nachman [34 and Brown and Uhlmann [13] proved uniqueness results for conductivities which are in $W^{2, p}$ with $p>1$ and $W^{1, p}$ with $p>2$, respectively. Finally, in 2006 the question (1.9) has been answered to be positive by Astala and Päivärinta [3] in dimension two. For surveys on the subject, we refer the reader to [10, 17, 20, 33, 43] and the references therein.

Although there exists a large number of papers on the numerical solution of the inverse problems of EIT, among these also papers considering the Kohn-Vogelius functional (see, e.g., 28, 29]) and total variation regularization (see, e.g., [21, 36]), we have not yet found investigations on the discretization error in a combination of both functionals for the fully nonlinear setting, a fact which motivated the research presented in this paper.

Throughout the paper we use the standard notion of Sobolev spaces $H^{1}(\Omega), H_{0}^{1}(\Omega), W^{k, p}(\Omega)$, etc from, for example, [1]. If not stated otherwise we write $\int_{\Omega} \cdots$ instead of $\int_{\Omega} \cdots d x$.

\section{Problem setting and preliminaries}

\section{$2.1 \quad$ Notations}

Let us denote by

$$
\gamma: H^{1}(\Omega) \rightarrow H^{1 / 2}(\partial \Omega)
$$

the continuous Dirichlet trace operator while

$$
\gamma^{-1}: H^{1 / 2}(\partial \Omega) \rightarrow H^{1}(\Omega)
$$

is the continuous right inverse operator of $\gamma$, i.e. $\left(\gamma \circ \gamma^{-1}\right) g=g$ for all $g \in H^{1 / 2}(\partial \Omega)$. With $f \in H^{-1}(\Omega)$ (with a slight abuse of notation) in (1.1) being given, let us denote

$$
c_{f}:=(f, 1),
$$

where the expression $(f, \varphi)$ denotes the value of the functional $f \in H^{-1}(\Omega)$ at $\varphi \in H^{1}(\Omega)$. We also denote

$$
H_{-c_{f}}^{-1 / 2}(\partial \Omega):=\left\{j \in H^{-1 / 2}(\partial \Omega) \mid\langle j, 1\rangle=-c_{f}\right\},
$$

where the notation $\langle j, g\rangle$ stands for the value of the functional $j \in H^{-1 / 2}(\partial \Omega)$ at $g \in H^{1 / 2}(\partial \Omega)$. Similarly, we denote

$$
H_{\diamond}^{1 / 2}(\partial \Omega):=\left\{g \in H^{1 / 2}(\partial \Omega) \mid \int_{\partial \Omega} g(s)=0\right\}
$$

while $H_{\diamond}^{1}(\Omega)$ is the closed subspace of $H^{1}(\Omega)$ consisting of all functions with zero mean on the boundary, i.e.

$$
H_{\diamond}^{1}(\Omega):=\left\{u \in H^{1}(\Omega) \mid \int_{\partial \Omega} \gamma u=0\right\} .
$$

Let us denote by $C_{\diamond}^{\Omega}$ the positive constant appearing in the Poincaré-Friedrichs inequality (see, for example, 38])

$$
C_{\diamond}^{\Omega} \int_{\Omega} \varphi^{2} \leq \int_{\Omega}|\nabla \varphi|^{2} \text { for all } \varphi \in H_{\diamond}^{1}(\Omega) .
$$

Then for all $q \in \mathcal{Q}$ defined by 1.4 , the coercivity condition

$$
\|\varphi\|_{H^{1}(\Omega)}^{2} \leq \frac{1+C_{\diamond}^{\Omega}}{C_{\diamond}^{\Omega}} \int_{\Omega}|\nabla \varphi|^{2} \leq \frac{1+C_{\diamond}^{\Omega}}{C_{\diamond}^{\Omega} \underline{q}} \int_{\Omega} q \nabla \varphi \cdot \nabla \varphi
$$


holds for all $\varphi \in H_{\diamond}^{1}(\Omega)$. Furthermore, since $H_{0}^{1}(\Omega):=\left\{u \in H^{1}(\Omega) \mid \gamma u=0\right\} \subset H_{\diamond}^{1}(\Omega)$, the inequality (2.2) remains valid for all $\varphi \in H_{0}^{1}(\Omega)$.

Finally, for the sake of completeness we briefly introduce the space of functions with bounded total variation; for more details one may consult [4, 24]. A scalar function $q \in L^{1}(\Omega)$ is said to be of bounded total variation if

$$
T V(q):=\int_{\Omega}|\nabla q|:=\sup \left\{\left.\int_{\Omega} q \operatorname{div} \Xi\left|\Xi \in C_{c}^{1}(\Omega)^{d},\right| \Xi(x)\right|_{\infty} \leq 1, x \in \Omega\right\}<\infty .
$$

Here $|\cdot|_{\infty}$ denotes the $\ell_{\infty}$-norm on $\mathbb{R}^{d}$ defined by $|x|_{\infty}=\max _{1 \leq i \leq d}\left|x_{i}\right|$ and $C_{c}^{1}(\Omega)$ the space of continuously differentiable functions with compact support in $\Omega$. The space of all functions in $L^{1}(\Omega)$ with bounded total variation is denoted by

$$
B V(\Omega)=\left\{q \in L^{1}(\Omega)\left|\int_{\Omega}\right| \nabla q \mid<\infty\right\}
$$

which is a Banach space with the norm

$$
\|q\|_{B V(\Omega)}:=\|q\|_{L^{1}(\Omega)}+\int_{\Omega}|\nabla q| .
$$

Furthermore, if $\Omega$ is an open bounded set with Lipschitz boundary, then $W^{1,1}(\Omega) \varsubsetneqq B V(\Omega)$.

\subsection{Neumann operator, Dirichlet operator and Neumann-to-Dirichlet map}

\subsubsection{Neumann operator}

We consider the following Neumann problem

$$
-\nabla \cdot(q \nabla u)=f \text { in } \Omega \text { and } q \nabla u \cdot \vec{n}=j \text { on } \partial \Omega .
$$

By the coercivity condition $(2.2)$ and the Riesz representation theorem, we conclude that for each $q \in \mathcal{Q}$ and $j \in H_{-c_{f}}^{-1 / 2}(\partial \Omega)$ there exists a unique weak solution $u$ of the problem 2.3 in the sense that $u \in H_{\diamond}^{1}(\Omega)$ and satisfies the identity

$$
\int_{\Omega} q \nabla u \cdot \nabla \varphi=\langle j, \gamma \varphi\rangle+(f, \varphi)
$$

for all $\varphi \in H_{\diamond}^{1}(\Omega)$. By the imposed compatibility condition $\langle j, 1\rangle=-c_{f}$, i.e.

$$
\langle j, 1\rangle+(f, 1)=0,
$$

and the fact that $H^{1}(\Omega)=H_{\diamond}^{1}(\Omega)+\operatorname{span}\{1\}$, equation 2.4 is satisfied for all $\varphi \in H^{1}(\Omega)$. Furthermore, this solution satisfies the following estimate

$$
\begin{aligned}
\|u\|_{H^{1}(\Omega)} & \leq \frac{1+C_{\diamond}^{\Omega}}{C_{\diamond}^{\Omega} \underline{q}}\left(\|\gamma\|_{\mathcal{L}\left(H^{1}(\Omega), H^{1 / 2}(\partial \Omega)\right.}\|j\|_{H^{-1 / 2}(\partial \Omega)}+\|f\|_{H^{-1}(\Omega)}\right) \\
& \leq C_{\mathcal{N}}\left(\|j\|_{H^{-1 / 2}(\partial \Omega)}+\|f\|_{H^{-1}(\Omega)}\right)
\end{aligned}
$$

where

$$
C_{\mathcal{N}}:=\frac{1+C_{\diamond}^{\Omega}}{C_{\diamond}^{\Omega} \underline{q}} \max \left(1,\|\gamma\|_{\mathcal{L}\left(H^{1}(\Omega), H^{1 / 2}(\partial \Omega)\right)}\right) .
$$

Then for any fixed $j \in H_{-c_{f}}^{-1 / 2}(\partial \Omega)$ we can define the Neumann operator

$$
\mathcal{N}: \mathcal{Q} \rightarrow H_{\diamond}^{1}(\Omega) \text { with } q \mapsto \mathcal{N}_{q} j
$$

which maps each $q \in \mathcal{Q}$ to the unique weak solution $\mathcal{N}_{q} j:=u$ of the problem 2.3.

Remark 2.1. We note that the restriction $j \in H_{-c_{f}}^{-1 / 2}(\partial \Omega)$ instead of $j \in H^{-1 / 2}(\partial \Omega)$ preserves the compatibility condition 2.5) for the pure Neumann problem. In case this condition fails, the strong form of the problem 2.3 has no solution. This is the reason why we require $j \in H_{-c_{f}}^{-1 / 2}(\partial \Omega)$. However, its weak form, i.e. the variational equation (2.4), attains a unique solution independently of the compatibility condition. By working with the weak form only, all results in the present paper remain valid for $j \in H^{-1 / 2}(\partial \Omega)$. 


\subsubsection{Dirichlet operator}

We now consider the following Dirichlet problem

$$
-\nabla \cdot(q \nabla v)=f \text { in } \Omega \text { and } v=g \text { on } \partial \Omega
$$

For each $q \in \mathcal{Q}$ and $g \in H^{1 / 2}(\partial \Omega)$, by the coercivity condition (2.2), the problem 2.7) attains a unique weak solution $v$ in the sense that $v \in H^{1}(\Omega), \gamma v=g$ and satisfies the identity

$$
\int_{\Omega} q \nabla v \cdot \nabla \psi=(f, \psi)
$$

for all $\psi \in H_{0}^{1}(\Omega)$. We can rewrite

$$
v=v_{0}+G,
$$

where $G=\gamma^{-1} g$ and $v_{0} \in H_{0}^{1}(\Omega)$ is the unique solution to the following variational problem

$$
\int_{\Omega} q \nabla v_{0} \cdot \nabla \psi=(f, \psi)-\int_{\Omega} q \nabla G \cdot \nabla \psi
$$

for all $\psi \in H_{0}^{1}(\Omega)$. Since

$$
\|G\|_{H^{1}(\Omega)} \leq\left\|\gamma^{-1}\right\|_{\mathcal{L}\left(H^{1 / 2}(\partial \Omega), H^{1}(\Omega)\right.}\|g\|_{H^{1 / 2}(\partial \Omega)},
$$

we thus obtain the priori estimate

$$
\begin{aligned}
\|v\|_{H^{1}(\Omega)} & \leq\left\|v_{0}\right\|_{H^{1}(\Omega)}+\|G\|_{H^{1}(\Omega)} \\
& \leq \frac{1+C_{\diamond}^{\Omega}}{C_{\diamond}^{\Omega} \underline{q}}\|f\|_{H^{-1}(\Omega)}+\frac{1+C_{\diamond}^{\Omega}}{C_{\diamond}^{\Omega} \underline{q}}\|G\|_{H^{1}(\Omega)}+\|G\|_{H^{1}(\Omega)} \\
& \leq C_{\mathcal{D}}\left(\|g\|_{H^{1 / 2}(\partial \Omega)}+\|f\|_{H^{-1}(\Omega)}\right)
\end{aligned}
$$

where

$$
\left.C_{\mathcal{D}}:=\max \left(\frac{1+C_{\diamond}^{\Omega}}{C_{\diamond}^{\Omega} \underline{q}},\left(\frac{1+C_{\diamond}^{\Omega}}{C_{\diamond}^{\Omega} \underline{q}} \bar{q}+1\right)\left\|\gamma^{-1}\right\|_{\mathcal{L}\left(H^{1 / 2}(\partial \Omega), H^{1}(\Omega)\right.}\right)\right) .
$$

The Dirichlet operator is for any fixed $g \in H^{1 / 2}(\partial \Omega)$ defined as

$$
\mathcal{D}: \mathcal{Q} \rightarrow H^{1}(\Omega) \text { with } q \mapsto \mathcal{D}_{q} g
$$

which maps each $q \in \mathcal{Q}$ to the unique weak solution $\mathcal{D}_{q} g:=v$ of the problem (2.7).

\subsubsection{Neumann-to-Dirichlet map}

For any fixed $q \in \mathcal{Q}$ we can define the Neumann-to-Dirichlet map

$$
\begin{aligned}
\Lambda_{q}: H_{-c_{f}}^{-1 / 2}(\partial \Omega) & \rightarrow H_{\diamond}^{1 / 2}(\partial \Omega) \\
j & \mapsto \Lambda_{q} j:=\gamma \mathcal{N}_{q} j .
\end{aligned}
$$

Since

$$
\int_{\Omega} q \nabla \mathcal{N}_{q} j \cdot \nabla \psi=(f, \psi)
$$

for all $\psi \in H_{0}^{1}(\Omega)$, in view of $(2.8)$ we conclude that

$$
\Lambda_{q} j=g \text { if and only if } \mathcal{N}_{q} j=\mathcal{D}_{q} g \text {. }
$$




\subsection{Identification problem}

The inverse problem is stated as follows.

$$
\text { Given } f \in H^{-1}(\Omega),\left(j^{\dagger}, g^{\dagger}\right) \in H_{-c_{f}}^{-1 / 2}(\partial \Omega) \times H_{\diamond}^{1 / 2}(\partial \Omega) \text { with } \Lambda_{q^{\dagger}} j^{\dagger}=g^{\dagger} \text {, find } q^{\dagger} \in \mathcal{Q} \text {. }
$$

In other words, the problem of interest is, given $f \in H^{-1}(\Omega)$, and a single Neumann-Dirichlet pair $\left(j^{\dagger}, g^{\dagger}\right) \in$ $H_{-c_{f}}^{-1 / 2}(\partial \Omega) \times H_{\diamond}^{1 / 2}(\partial \Omega)$, to find $q^{\dagger} \in \mathcal{Q}$ and $\Phi^{\dagger} \in H_{\diamond}^{1}(\Omega)$ such that the system 1.1 1.3$)$ is satisfied in the weak sense.

\subsection{Total variation regularization}

Assume that $\left(j_{\delta}, g_{\delta}\right) \in H_{-c_{f}}^{-1 / 2}(\partial \Omega) \times H_{\diamond}^{1 / 2}(\partial \Omega)$ is the measured data of the exact boundary values $\left(j^{\dagger}, g^{\dagger}\right)$ with

$$
\left\|j_{\delta}-j^{\dagger}\right\|_{H^{-1 / 2}(\partial \Omega)}+\left\|g_{\delta}-g^{\dagger}\right\|_{H^{1 / 2}(\partial \Omega)} \leq \delta
$$

for some measurement error $\delta>0$. Our problem is now to reconstruct the conductivity $q^{\dagger} \in \mathcal{Q}$ from this perturbed data $\left(j_{\delta}, g_{\delta}\right)$. For this purpose we consider the cost functional

$$
\mathcal{J}_{\delta}(q):=\int_{\Omega} q \nabla\left(\mathcal{N}_{q} j_{\delta}-\mathcal{D}_{q} g_{\delta}\right) \cdot \nabla\left(\mathcal{N}_{q} j_{\delta}-\mathcal{D}_{q} g_{\delta}\right),
$$

where $\mathcal{N}_{q} j_{\delta}$ and $\mathcal{D}_{q} g_{\delta}$ is the unique weak solutions of the problems (2.3) and (2.7), respectively, with $j$ in (2.3) and $g$ in 2.7) being replaced by $j_{\delta}$ and $g_{\delta}$. Furthermore, to estimate the possibly discontinuous conductivity, we here use the total variation regularization (cf., e.g., 14, 21, 22]), i.e., we consider the minimization problem

$$
\min _{q \in \mathcal{Q}_{a d}} \Upsilon_{\rho, \delta}(q):=\min _{q \in \mathcal{Q}_{a d}}\left(\mathcal{J}_{\delta}(q)+\rho \int_{\Omega}|\nabla q|\right),
$$

where

$$
\mathcal{Q}_{a d}:=\mathcal{Q} \cap B V(\Omega)
$$

is the admissible set of the sought conductivities.

Remark 2.2. The noise model (2.11) is to some extent an idealized one, since in practice, measurement precision might be different for the current $j$ and the voltage $g$, and, more importantly, it will be first of all be given with respect to some $L^{p}$ norm (e.g., $p=2$ corresponding to normally and $p=\infty$ to uniformly distributed noise) rather than in $H^{-1 / 2}(\partial \Omega) \times H^{1 / 2}(\partial \Omega)$. While the Neumann data part is unproblematic, by continuity of the embedding of $L^{p}(\partial \Omega)$ in $H^{-1 / 2}(\partial \Omega)$ for $p \geq 2 \frac{d-1}{d}$, we can obtain an $H^{1 / 2}(\partial \Omega)$ version of the originally $L^{p}(\partial \Omega)$ Dirichlet data e.g. by Tikhonov regularization (cf. [22] and the references therein) as follows. For simplicity, we restrict ourselves to the Hilbert space case $p=2$ and assume that we have measurements $\tilde{g}_{\delta_{g}} \in L^{2}(\partial \Omega)$ such that

$$
\left\|\tilde{g}_{\delta_{g}}-g^{\dagger}\right\|_{L^{2}(\partial \Omega)} \leq \delta_{g}
$$

Tikhonov regularization applied to the embedding operator $K: H^{1 / 2}(\partial \Omega) \rightarrow L^{2}(\partial \Omega)$ amounts to finding a minimizer $g_{\alpha}^{\delta_{g}}$ of

$$
\min _{g \in H^{1 / 2}(\partial \Omega)}\left\|K g-\tilde{g}_{\delta_{g}}\right\|_{L^{2}(\partial \Omega)}^{2}+\alpha\|g\|_{H^{1 / 2}(\partial \Omega)}^{2}
$$

where we use

$$
\|g\|_{H^{1 / 2}(\partial \Omega)}:=\left\|\gamma^{-1} g\right\|_{H^{1}(\Omega)}=\left(\int_{\Omega}\left(\left|\nabla \gamma^{-1} g\right|^{2}+\left|\gamma^{-1} g\right|^{2}\right) d x\right)^{1 / 2}
$$

as a norm on $H^{1 / 2}(\partial \Omega)$. The first order optimality conditions for this quadratic minimization problem yield

$$
\int_{\partial \Omega} \phi\left(g_{\alpha}^{\delta_{g}}-\tilde{g}_{\delta_{g}}\right) d s+\alpha \int_{\Omega}\left(\nabla \gamma^{-1} g_{\alpha}^{\delta_{g}} \cdot \nabla \gamma^{-1} \phi+\gamma^{-1} g_{\alpha}^{\delta_{g}} \gamma^{-1} \phi\right) d x=0 \text { for all } \phi \in H^{1 / 2}(\partial \Omega),
$$


which is equivalent to

$$
\int_{\partial \Omega} \gamma \varphi\left(\gamma w-\tilde{g}_{\delta_{g}}\right) d s+\alpha \int_{\Omega}(\nabla w \cdot \nabla \varphi+w \varphi) d x=0 \text { for all } \varphi \in H^{1}(\Omega),
$$

for $w=\gamma^{-1} g_{\alpha}^{\delta_{g}}$, i.e., the weak form of the Robin problem

$$
\begin{cases}-\Delta w+w & =0 \text { in } \Omega, \\ \alpha \nabla w \cdot \vec{n}+w & =\tilde{g}_{\delta_{g}} \text { on } \partial \Omega .\end{cases}
$$

Thus, according to well-known results from regularization theory (cf., e.g. 22]), the smoothed version $g_{\delta}:=$ $g_{\alpha}^{\delta_{g}}=\gamma w$ (where $w$ weakly solves 2.13) of $\tilde{g}_{\delta_{g}}$ converges to $g^{\dagger}$ as $\delta_{g}$ tends to zero, provided the regularization parameter $\alpha=\alpha\left(\delta_{g}, \tilde{g}_{\delta_{g}}\right)$ is chosen appropriately. The latter can, e.g., be done by the discrepancy principle, where $\alpha$ is chosen such that

$$
\left\|K g_{\alpha}^{\delta_{g}}-\tilde{g}_{\delta_{g}}\right\|_{L^{2}(\partial \Omega)}^{2}=\int_{\partial \Omega}\left|g_{\alpha}^{\delta_{g}}-\tilde{g}_{\delta_{g}}\right|^{2} d x \sim \delta_{g}^{2}
$$

We also wish to mention the complete electrode model cf., e.g., [40, which fully takes into account the fact that current and voltage are typically not measured pointwise on the whole boundary, but via a set of finitely many electrodes with finite geometric extensions as well as contact impedances.

\subsection{Auxiliary results}

Now we summarize some useful properties of the Neumann and Dirichlet operators. The proof of the following result is based on standard arguments and therefore omitted.

Lemma 2.3. Let $(j, g) \in H_{-c_{f}}^{-1 / 2}(\partial \Omega) \times H_{\diamond}^{1 / 2}(\partial \Omega)$ be fixed.

(i) The Neumann operator $\mathcal{N}: \mathcal{Q} \subset L^{\infty}(\Omega) \rightarrow H_{\diamond}^{1}(\Omega)$ is continuously Fréchet differentiable on the set $\mathcal{Q}$. For each $q \in \mathcal{Q}$ the action of the Fréchet derivative in direction $\xi \in L^{\infty}(\Omega)$ denoted by $\eta_{\mathcal{N}}:=\mathcal{N}_{q}^{\prime} j(\xi):=\mathcal{N}^{\prime}(q) \xi$ is the unique weak solution in $H_{\diamond}^{1}(\Omega)$ to the Neumann problem

$$
-\nabla \cdot\left(q \nabla \eta_{\mathcal{N}}\right)=\nabla \cdot\left(\xi \nabla \mathcal{N}_{q} j\right) \text { in } \Omega \text { and } q \nabla \eta_{\mathcal{N}} \cdot \vec{n}=-\xi \nabla \mathcal{N}_{q} j \cdot \vec{n} \text { on } \partial \Omega
$$

in the sense that the identity

$$
\int_{\Omega} q \nabla \eta_{\mathcal{N}} \cdot \nabla \varphi=-\int_{\Omega} \xi \nabla \mathcal{N}_{q} j \cdot \nabla \varphi
$$

holds for all $\varphi \in H_{\diamond}^{1}(\Omega)$. Furthermore, the following estimate is fulfilled

$$
\left\|\eta_{\mathcal{N}}\right\|_{H^{1}(\Omega)} \leq \frac{\left(1+C_{\diamond}^{\Omega}\right) C_{\mathcal{N}}}{C_{\diamond}^{\Omega} \underline{q}}\left(\|j\|_{H^{-1 / 2}(\partial \Omega)}+\|f\|_{H^{-1}(\Omega)}\right)\|\xi\|_{L^{\infty}(\Omega)} .
$$

(ii) The Dirichlet operator $\mathcal{D}: \mathcal{Q} \subset L^{\infty}(\Omega) \rightarrow H_{\diamond}^{1}(\Omega)$ is continuously Fréchet differentiable on the set $\mathcal{Q}$. For each $q \in \mathcal{Q}$ the action of the Fréchet derivative in direction $\xi \in L^{\infty}(\Omega)$ denoted by $\eta_{\mathcal{D}}:=\mathcal{D}_{q}^{\prime} g(\xi)=: \mathcal{D}^{\prime}(q) \xi$ is the unique weak solution in $H_{0}^{1}(\Omega)$ to the Dirichlet problem

$$
-\nabla \cdot\left(q \nabla \eta_{\mathcal{D}}\right)=\nabla \cdot\left(\xi \nabla \mathcal{D}_{q} g\right) \text { in } \Omega \text { and } \eta_{\mathcal{D}}=0 \text { on } \partial \Omega
$$

in the sense that it satisfies the equation

$$
\int_{\Omega} q \nabla \eta_{\mathcal{D}} \cdot \nabla \psi=-\int_{\Omega} \xi \nabla \mathcal{D}_{q} g \cdot \nabla \psi
$$

for all $\psi \in H_{0}^{1}(\Omega)$. Furthermore, the following estimate is fulfilled

$$
\left\|\eta_{\mathcal{D}}\right\|_{H^{1}(\Omega)} \leq \frac{\left(1+C_{\diamond}^{\Omega}\right) C_{\mathcal{D}}}{C_{\diamond}^{\Omega} \underline{q}}\left(\|g\|_{H^{1 / 2}(\partial \Omega)}+\|f\|_{H^{-1}(\Omega)}\right)\|\xi\|_{L^{\infty}(\Omega)} .
$$


Lemma 2.4. If the sequence $\left(q_{n}\right) \subset \mathcal{Q}$ converges to $q$ in the $L^{1}(\Omega)$-norm, then $q \in \mathcal{Q}$ and for any fixed $\left(j_{\delta}, g_{\delta}\right) \in H_{-c_{f}}^{-1 / 2}(\partial \Omega) \times H_{\diamond}^{1 / 2}(\partial \Omega)$ the sequence $\left(\mathcal{N}_{q_{n}} j_{\delta}, \mathcal{D}_{q_{n}} g_{\delta}\right)$ converges to $\left(\mathcal{N}_{q} j_{\delta}, \mathcal{D}_{q} g_{\delta}\right)$ in the $H^{1}(\Omega) \times$ $H^{1}(\Omega)$-norm. Furthermore, there holds

$$
\lim _{n \rightarrow \infty} \mathcal{J}_{\delta}\left(q_{n}\right)=\mathcal{J}_{\delta}(q)
$$

where the functional $\mathcal{J}_{\delta}$ is defined in 2.12 .

Proof. Since $\left(q_{n}\right) \subset \mathcal{Q}$ converges to $q$ in the $L^{1}(\Omega)$-norm, up to a subsequence we assume that it converges to $q$ a.e. in $\Omega$, which implies that $q \in \mathcal{Q}$. For all $\varphi \in H_{\diamond}^{1}(\Omega)$ we infer from 2.4 that

$$
\int_{\Omega} q_{n} \nabla \mathcal{N}_{q_{n}} j_{\delta} \cdot \nabla \varphi=\left\langle j_{\delta}, \gamma \varphi\right\rangle+(f, \varphi)=\int_{\Omega} q \nabla \mathcal{N}_{q} j_{\delta} \cdot \nabla \varphi
$$

and so that

$$
\int_{\Omega} q_{n} \nabla\left(\mathcal{N}_{q_{n}} j_{\delta}-\mathcal{N}_{q} j_{\delta}\right) \cdot \nabla \varphi=\int_{\Omega}\left(q-q_{n}\right) \nabla \mathcal{N}_{q} j_{\delta} \cdot \nabla \varphi
$$

Taking $\varphi=\mathcal{N}_{q_{n}} j_{\delta}-\mathcal{N}_{q} j_{\delta}$, by 2.2 , we get

$$
\begin{aligned}
\frac{C_{\diamond}^{\Omega} \underline{q}}{1+C_{\diamond}^{\Omega}}\left\|\mathcal{N}_{q_{n}} j_{\delta}-\mathcal{N}_{q} j_{\delta}\right\|_{H^{1}(\Omega)}^{2} & \leq \int_{\Omega} q_{n} \nabla\left(\mathcal{N}_{q_{n}} j_{\delta}-\mathcal{N}_{q} j_{\delta}\right) \cdot \nabla\left(\mathcal{N}_{q_{n}} j_{\delta}-\mathcal{N}_{q} j_{\delta}\right) \\
& =\int_{\Omega}\left(q-q_{n}\right) \nabla \mathcal{N}_{q} j_{\delta} \cdot \nabla\left(\mathcal{N}_{q_{n}} j_{\delta}-\mathcal{N}_{q} j_{\delta}\right) \\
& \leq\left(\int_{\Omega}\left|q-q_{n}\right|^{2}\left|\nabla \mathcal{N}_{q} j_{\delta}\right|^{2}\right)^{1 / 2}\left(\int_{\Omega}\left|\nabla\left(\mathcal{N}_{q_{n}} j_{\delta}-\mathcal{N}_{q} j_{\delta}\right)\right|^{2}\right)^{1 / 2}
\end{aligned}
$$

and so that

$$
\left\|\mathcal{N}_{q_{n}} j_{\delta}-\mathcal{N}_{q} j_{\delta}\right\|_{H^{1}(\Omega)} \leq \frac{1+C_{\diamond}^{\Omega}}{C_{\diamond}^{\Omega} \underline{q}}\left(\int_{\Omega}\left|q-q_{n}\right|^{2}\left|\nabla \mathcal{N}_{q} j_{\delta}\right|^{2}\right)^{1 / 2} .
$$

Hence, by the Lebesgue dominated convergence theorem, we deduce from the last inequality that

$$
\lim _{n \rightarrow \infty}\left\|\mathcal{N}_{q_{n}} j_{\delta}-\mathcal{N}_{q} j_{\delta}\right\|_{H^{1}(\Omega)}=0 .
$$

Similarly to 2.16, we also get

$$
\int_{\Omega} q_{n} \nabla\left(\mathcal{D}_{q_{n}} g_{\delta}-\mathcal{D}_{q} g_{\delta}\right) \cdot \nabla \psi=\int_{\Omega}\left(q-q_{n}\right) \nabla \mathcal{D}_{q} g_{\delta} \cdot \nabla \psi
$$

for all $\psi \in H_{0}^{1}(\Omega)$. Since $\gamma \mathcal{D}_{q_{n}} g_{\delta}=\gamma \mathcal{D}_{q} g_{\delta}=g_{\delta}$, taking $\psi=\mathcal{D}_{q_{n}} g_{\delta}-\mathcal{D}_{q} g_{\delta} \in H_{0}^{1}(\Omega)$ in the last equation, we also obtain the limit

$$
\lim _{n \rightarrow \infty}\left\|\mathcal{D}_{q_{n}} g_{\delta}-\mathcal{D}_{q} g_{\delta}\right\|_{H^{1}(\Omega)}=0 .
$$

Next, we rewrite the functional $\mathcal{J}_{\delta}$ as follows

$$
\begin{aligned}
\mathcal{J}_{\delta}\left(q_{n}\right) & =\int_{\Omega} q_{n} \nabla \mathcal{N}_{q_{n}} j_{\delta} \cdot \nabla \mathcal{N}_{q_{n}} j_{\delta}-2 \int_{\Omega} q_{n} \nabla \mathcal{N}_{q_{n}} j_{\delta} \cdot \nabla \mathcal{D}_{q_{n}} g_{\delta}+\int_{\Omega} q_{n} \nabla \mathcal{D}_{q_{n}} g_{\delta} \cdot \nabla \mathcal{D}_{q_{n}} g_{\delta} \\
& =\left\langle j_{\delta}, \gamma \mathcal{N}_{q_{n}} j_{\delta}\right\rangle+\left(f, \mathcal{N}_{q_{n}} j_{\delta}\right)-2\left(\left\langle j_{\delta}, g_{\delta}\right\rangle+\left(f, \mathcal{D}_{q_{n}} g_{\delta}\right)\right)+\int_{\Omega} q_{n} \nabla \mathcal{D}_{q_{n}} g_{\delta} \cdot \nabla \mathcal{D}_{q_{n}} g_{\delta}
\end{aligned}
$$

and, by (2.17)-2.18), have that

$$
\left\langle j_{\delta}, \gamma \mathcal{N}_{q_{n}} j_{\delta}\right\rangle+\left(f, \mathcal{N}_{q_{n}} j_{\delta}-2 \mathcal{D}_{q_{n}} g_{\delta}\right) \rightarrow\left\langle j_{\delta}, \gamma \mathcal{N}_{q} j_{\delta}\right\rangle+\left(f, \mathcal{N}_{q} j_{\delta}-2 \mathcal{D}_{q} g_{\delta}\right)
$$


as $n$ tends to $\infty$. We now consider the difference

$$
\begin{aligned}
\int_{\Omega} q_{n} \nabla \mathcal{D}_{q_{n}} g_{\delta} \cdot \nabla \mathcal{D}_{q_{n}} g_{\delta} & -\int_{\Omega} q \nabla \mathcal{D}_{q} g_{\delta} \cdot \nabla \mathcal{D}_{q} g_{\delta} \\
& =\int_{\Omega} q_{n} \nabla\left(\mathcal{D}_{q_{n}} g_{\delta}-\mathcal{D}_{q} g_{\delta}\right) \cdot \nabla\left(\mathcal{D}_{q_{n}} g_{\delta}+\mathcal{D}_{q} g_{\delta}\right)-\int_{\Omega}\left(q-q_{n}\right) \nabla \mathcal{D}_{q} g_{\delta} \cdot \nabla \mathcal{D}_{q} g_{\delta}
\end{aligned}
$$

and note that

$$
\int_{\Omega}\left(q-q_{n}\right) \nabla \mathcal{D}_{q} g_{\delta} \cdot \nabla \mathcal{D}_{q} g_{\delta} \rightarrow 0
$$

as $n$ goes to $\infty$, by the Lebesgue dominated convergence theorem. Furthermore, then applying the CauchySchwarz inequality, we also get that

$$
\begin{aligned}
\mid \int_{\Omega} q_{n} & \nabla\left(\mathcal{D}_{q_{n}} g_{\delta}-\mathcal{D}_{q} g_{\delta}\right) \cdot \nabla\left(\mathcal{D}_{q_{n}} g_{\delta}+\mathcal{D}_{q} g_{\delta}\right) \mid \\
\leq & \bar{q}\left(\int_{\Omega}\left|\nabla\left(\mathcal{D}_{q_{n}} g_{\delta}-\mathcal{D}_{q} g_{\delta}\right)\right|^{2}\right)^{1 / 2}\left(\int_{\Omega}\left|\nabla\left(\mathcal{D}_{q_{n}} g_{\delta}+\mathcal{D}_{q} g_{\delta}\right)\right|^{2}\right)^{1 / 2} \\
\leq & \bar{q}\left\|\mathcal{D}_{q_{n}} g_{\delta}-\mathcal{D}_{q} g_{\delta}\right\|_{H^{1}(\Omega)}\left(\left\|\mathcal{D}_{q_{n}} g_{\delta}\right\|_{H^{1}(\Omega)}+\left\|\mathcal{D}_{q} g_{\delta}\right\|_{H^{1}(\Omega)}\right) \rightarrow 0
\end{aligned}
$$

as $n$ approaches $\infty$, here we used 2.10 and 2.18. We thus obtain that

$$
\int_{\Omega} q_{n} \nabla \mathcal{D}_{q_{n}} g_{\delta} \cdot \nabla \mathcal{D}_{q_{n}} g_{\delta} \rightarrow \int_{\Omega} q \nabla \mathcal{D}_{q} g_{\delta} \cdot \nabla \mathcal{D}_{q} g_{\delta}
$$

as $n$ tends to $\infty$. Then we deduce from $2.19-2.21$ that

$$
\begin{aligned}
\lim _{n \rightarrow \infty} \mathcal{J}_{\delta}\left(q_{n}\right) & =\left\langle j_{\delta}, \gamma \mathcal{N}_{q} j_{\delta}\right\rangle+\left(f, \mathcal{N}_{q} j_{\delta}\right)-2\left\langle j_{\delta}, g_{\delta}\right\rangle-2\left(f, \mathcal{D}_{q} g_{\delta}\right)+\int_{\Omega} q \nabla \mathcal{D}_{q} g_{\delta} \cdot \nabla \mathcal{D}_{q} g_{\delta} \\
& =\int_{\Omega} q \nabla \mathcal{N}_{q} j_{\delta} \cdot \nabla \mathcal{N}_{q} j_{\delta}-2 \int_{\Omega} q \nabla \mathcal{N}_{q} j_{\delta} \cdot \nabla \mathcal{D}_{q} g_{\delta}+\int_{\Omega} q \nabla \mathcal{D}_{q} g_{\delta} \cdot \nabla \mathcal{D}_{q} g_{\delta} \\
& =\mathcal{J}_{\delta}(q),
\end{aligned}
$$

which finishes the proof.

Lemma 2.5 ([24]). (i) Let $\left(q_{n}\right)$ be a bounded sequence in the $B V(\Omega)$-norm. Then a subsequence which is denoted by the same symbol and an element $q \in B V(\Omega)$ exist such that $\left(q_{n}\right)$ converges to $q$ in the $L^{1}(\Omega)$-norm.

(ii) Let $\left(q_{n}\right)$ be a sequence in $B V(\Omega)$ converging to $q$ in the $L^{1}(\Omega)$-norm. Then $q \in B V(\Omega)$ and

$$
\int_{\Omega}|\nabla q| \leq \liminf _{n \rightarrow \infty} \int_{\Omega}\left|\nabla q_{n}\right|
$$

We mention that equality need not be achieved in 2.22 . Here is a counterexample from 24 . Let $\Omega=(0,2 \pi)$ and $q_{n}(x)=\frac{1}{n} \sin n x$ for $x \in \Omega$ and $n \in \mathbb{N}$. Then $\left\|q_{n}\right\|_{L^{1}(\Omega)} \rightarrow 0$ as $n \rightarrow \infty$, but $\int_{\Omega}\left|\nabla q_{n}\right|=4$ for each $n \in \mathbb{N}$. Let us quote the following useful result on approximation of $B V$-functions by smooth functions.

Lemma 2.6 ([5, 16]). Assume that $w \in B V(\Omega)$. Then for all $\alpha>0$ an element $w^{\alpha} \in C^{\infty}(\Omega)$ exists such that

$$
\int_{\Omega}\left|w-w^{\alpha}\right| \leq \alpha \int_{\Omega}|\nabla w|, \quad \int_{\Omega}\left|\nabla w^{\alpha}\right| \leq(1+C \alpha) \int_{\Omega}|\nabla w| \text { and } \int_{\Omega}\left|D^{2} w^{\alpha}\right| \leq C \alpha^{-1} \int_{\Omega}|\nabla w|,
$$

where the positive constant $C$ is independent of $\alpha$.

Now, we are in a position to prove the main result of this section

Theorem 2.7. The problem $\left(\mathcal{P}_{\rho, \delta}\right)$ attains a solution $q_{\rho, \delta}$, which is called the regularized solution of the identification problem. 
Proof. Let $\left(q_{n}\right) \subset \mathcal{Q}_{a d}$ be a minimizing sequence of the problem $\left(\mathcal{P}_{\rho, \delta}\right)$, i.e.,

$$
\lim _{n \rightarrow \infty}\left(\mathcal{J}_{\delta}\left(q_{n}\right)+\rho \int_{\Omega}\left|\nabla q_{n}\right|\right)=\inf _{q \in \mathcal{Q}_{a d}}\left(\mathcal{J}_{\delta}(q)+\rho \int_{\Omega}|\nabla q|\right) .
$$

Then, due to Lemma 2.5 a subsequence which is not relabelled and an element $q \in \mathcal{Q}_{a d}$ exist such that $\left(q_{n}\right)$ converges to $q$ in the $L^{1}(\Omega)$-norm and

$$
\int_{\Omega}|\nabla q| \leq \liminf _{n \rightarrow \infty} \int_{\Omega}\left|\nabla q_{n}\right|
$$

Using Lemma 2.4 and by (2.23)-2.24, we obtain that

$$
\begin{aligned}
\mathcal{J}_{\delta}(q)+\rho \int_{\Omega}|\nabla q| & \leq \lim _{n \rightarrow \infty} \mathcal{J}_{\delta}\left(q_{n}\right)+\liminf _{n \rightarrow \infty} \rho \int_{\Omega}\left|\nabla q_{n}\right| \\
& =\liminf _{n \rightarrow \infty}\left(\mathcal{J}_{\delta}\left(q_{n}\right)+\rho \int_{\Omega}\left|\nabla q_{n}\right|\right) \\
& =\inf _{q \in \mathcal{Q}_{a d}}\left(\mathcal{J}_{\delta}(q)+\rho \int_{\Omega}|\nabla q|\right) .
\end{aligned}
$$

This means that $q$ is a solution of the problem $\left(\mathcal{P}_{\rho, \delta}\right)$, which finishes the proof.

\section{Finite element method for the identification problem}

Let $\left(\mathcal{T}^{h}\right)_{0<h<1}$ be a family of regular and quasi-uniform triangulations of the domain $\bar{\Omega}$ with the mesh size $h$ such that each vertex of the polygonal boundary $\partial \Omega$ is a node of $\mathcal{T}^{h}$. For the definition of the discretization space of the state functions let us denote

$$
\mathcal{V}_{1}^{h}:=\left\{v^{h} \in C(\bar{\Omega}) \mid v^{h}{ }_{\mid T} \in \mathcal{P}_{1}(T), \forall T \in \mathcal{T}^{h}\right\}
$$

and

$$
\mathcal{V}_{1, \diamond}^{h}:=\mathcal{V}_{1}^{h} \cap H_{\diamond}^{1}(\Omega) \text { and } \mathcal{V}_{1,0}^{h}:=\mathcal{V}_{1}^{h} \cap H_{0}^{1}(\Omega) \subset \mathcal{V}_{1, \diamond}^{h},
$$

where $\mathcal{P}_{1}$ consists of all polynomial functions of degree less than or equal to 1 .

To go further, we introduce the following modified Clément's interpolation operator, see [19].

Lemma 3.1. An interpolation operator $\Pi_{\diamond}^{h}: L^{1}(\Omega) \rightarrow \mathcal{V}_{1, \diamond}^{h}$ exists such that

$$
\Pi_{\diamond}^{h} \varphi^{h}=\varphi^{h} \text { for all } \varphi^{h} \in \mathcal{V}_{1, \diamond}^{h} \text { and } \Pi_{\diamond}^{h}\left(H_{0}^{1}(\Omega)\right) \subset \mathcal{V}_{1,0}^{h} \subset \mathcal{V}_{1, \diamond}^{h} .
$$

Furthermore, it satisfies the properties

$$
\lim _{h \rightarrow 0}\left\|\vartheta-\Pi_{\diamond}^{h} \vartheta\right\|_{H^{1}(\Omega)}=0 \quad \text { for all } \vartheta \in H_{\diamond}^{1}(\Omega)
$$

and

$$
\left\|\vartheta-\Pi_{\diamond}^{h} \vartheta\right\|_{H^{1}(\Omega)} \leq C h\|\vartheta\|_{H^{2}(\Omega)} \text { for all } \vartheta \in H_{\diamond}^{1}(\Omega) \cap H^{2}(\Omega)
$$

with the positive constant $C$ being independent of $h$ and $\vartheta$.

Proof. It is well known (see [19] and some generalizations [6, 7, 39]) that there is an interpolation operator

$$
\Pi^{h}: L^{1}(\Omega) \rightarrow \mathcal{V}_{1}^{h} \text { with } \Pi^{h} \varphi^{h}=\varphi^{h} \text { for all } \varphi^{h} \in \mathcal{V}_{1}^{h} \text { and } \Pi^{h}\left(H_{0}^{1}(\Omega)\right) \subset \mathcal{V}_{1,0}^{h}
$$

which satisfies the following properties

$$
\lim _{h \rightarrow 0}\left\|\vartheta-\Pi^{h} \vartheta\right\|_{H^{1}(\Omega)}=0 \text { for all } \vartheta \in H^{1}(\Omega)
$$


and

$$
\left\|\vartheta-\Pi^{h} \vartheta\right\|_{H^{1}(\Omega)} \leq C h\|\vartheta\|_{H^{2}(\Omega)} \text { for all } \vartheta \in H^{2}(\Omega) .
$$

We then define for each $\vartheta \in L^{1}(\Omega)$

$$
\Pi_{\diamond}^{h} \vartheta:=\Pi^{h} \vartheta-\frac{1}{|\partial \Omega|} \int_{\partial \Omega} \gamma \Pi^{h} \vartheta \in \mathcal{V}_{1, \diamond}^{h} .
$$

Then $\Pi_{\diamond}^{h}\left(L^{1}(\Omega)\right) \subset \mathcal{V}_{1, \diamond}^{h}, \Pi_{\diamond}^{h} \varphi^{h}=\varphi^{h}$ for all $\varphi^{h} \in \mathcal{V}_{1, \diamond}^{h}$ and $\Pi_{\diamond}^{h}\left(H_{0}^{1}(\Omega)\right) \subset \mathcal{V}_{1,0}^{h}$. Furthermore, since $\nabla \Pi_{\diamond}^{h} \vartheta=$ $\nabla \Pi^{h} \vartheta$ for all $\vartheta \in L^{1}(\Omega)$, the properties (3.1), (3.2) are deduced from (3.3), (3.4), respectively. The proof is completed.

We remark that the operator $\Pi^{h}$ in the above proof satisfies the estimate $\left\|\vartheta-\Pi^{h} \vartheta\right\|_{H^{k}(\Omega)} \leq C h^{l-k}\|\vartheta\|_{H^{l}(\Omega)}$ for $0 \leq k \leq l \leq 2$ and $\vartheta \in H^{l}(\Omega)$ (see [19]) which implies that

$$
\left\|\Pi_{\diamond}^{h} \vartheta\right\|_{H^{1}(\Omega)} \leq C\|\vartheta\|_{H^{1}(\Omega)} \quad \text { for all } \vartheta \in H^{1}(\Omega),
$$

an estimate that is required for the proof of part (ii) of the following proposition.

Similarly to the continuous case we have the following result.

Proposition 3.2. (i) Let $q$ be in $\mathcal{Q}$ and $j$ be in $H_{-c_{f}}^{-1 / 2}(\partial \Omega)$. Then the variational equation

$$
\int_{\Omega} q \nabla u^{h} \cdot \nabla \varphi^{h}=\left\langle j, \gamma \varphi^{h}\right\rangle+\left(f, \varphi^{h}\right) \text { for all } \varphi^{h} \in \mathcal{V}_{1, \diamond}^{h}
$$

admits a unique solution $u^{h} \in \mathcal{V}_{1, \diamond}^{h}$. Furthermore, there holds

$$
\left\|u^{h}\right\|_{H^{1}(\Omega)} \leq C_{\mathcal{N}}\left(\|j\|_{H^{-1 / 2}(\partial \Omega)}+\|f\|_{H^{-1}(\Omega)}\right) .
$$

(ii) Let $q$ be in $\mathcal{Q}$ and $g$ be in $H_{\diamond}^{1 / 2}(\partial \Omega)$. Then the equation

$$
\int_{\Omega} q \nabla v^{h} \cdot \nabla \psi^{h}=\left(f, \psi^{h}\right) \text { for all } \psi^{h} \in \mathcal{V}_{1,0}^{h}
$$

with $\gamma v^{h}=\gamma\left(\Pi_{\diamond}^{h}\left(\gamma^{-1} g\right)\right)$ has a unique solution $v^{h} \in \mathcal{V}_{1, \diamond}^{h}$. Furthermore, the stability estimate

$$
\left\|v^{h}\right\|_{H^{1}(\Omega)} \leq \bar{C}_{\mathcal{D}}\left(\|g\|_{H^{1 / 2}(\partial \Omega)}+\|f\|_{H^{-1}(\Omega)}\right)
$$

is satisfied, where $\bar{C}_{\mathcal{D}}:=\max \left(\frac{1+C_{\diamond}^{\Omega}}{C_{\diamond}^{\Omega} \underline{q}},\left(\frac{1+C_{\diamond}^{\Omega}}{C_{\diamond}^{\Omega} \underline{q}} \bar{q}+1\right)\left\|\Pi_{\diamond}^{h}\right\|_{\mathcal{L}\left(H^{1}(\Omega), H^{1}(\Omega)\right)}\left\|\gamma^{-1}\right\|_{\mathcal{L}\left(H^{1 / 2}(\partial \Omega), H^{1}(\Omega)\right)}\right)$.

Let $u$ and $u^{h}$ be solutions to (2.4) and (3.6), respectively. Due to the standard theory of the finite element method (see, for example, [11, 18, ), the estimate

$$
\left\|u-u^{h}\right\|_{H^{1}(\Omega)} \leq C h\|u\|_{H^{2}(\Omega)}
$$

holds in case $u \in H^{2}(\Omega)$, where the positive constant $C$ is independent of $h$ and $u$.

Assume that $v$ and $v^{h}$ are the solutions to $(2.8)$ and (3.8), where $v \in H^{2}(\Omega)$, we then have (see, for example, [11, Section 5.4]) that

$$
\left\|v-v^{h}\right\|_{H^{1}(\Omega)} \leq \inf _{\psi^{h} \in \mathcal{V}_{1,0}^{h}}\left\|v-\gamma^{-1} g-\psi^{h}\right\|_{H^{1}(\Omega)}+2\left\|\gamma^{-1} g-\Pi_{\diamond}^{h}\left(\gamma^{-1} g\right)\right\|_{H^{1}(\Omega)} .
$$

Since $v \in H^{2}(\Omega)$, it follows that $g=\gamma v \in H^{3 / 2}(\Omega)$ and so $\gamma^{-1} g \in H^{2}(\Omega)$. Due to the approximation property of the finite dimensional spaces $\mathcal{V}_{1,0}^{h} \subset H_{0}^{1}(\Omega)$ (which states that $\inf _{\psi^{h} \in \mathcal{V}_{1,0}^{h}}\left\|\psi-\psi^{h}\right\|_{H^{1}(\Omega)} \leq C h\|\psi\|_{H^{2}(\Omega)}$ for each $\psi \in H^{2}(\Omega) \cap H_{0}^{1}(\Omega)$, where the constant $C$ is independent of $h$ and $\psi$ ) and (3.4), we deduce

$$
\left\|v-v^{h}\right\|_{H^{1}(\Omega)} \leq C h\left(\|v\|_{H^{2}(\Omega)}+\left\|\gamma^{-1} g\right\|_{H^{2}(\Omega)}\right) .
$$

We also mention that above we approximate the Dirichlet boundary condition $g$ by $g^{h}:=\gamma\left(\Pi_{\diamond}^{h}\left(\gamma^{-1} g\right)\right.$. There exist some different choices for the approximation $g^{h}$; for example, the $L^{2}$-projection of $g$ on the set $\mathcal{S}_{\partial \Omega}^{h}:=\left\{\gamma \varphi^{h} \mid \varphi^{h} \in \mathcal{V}_{1}^{h}\right\}$, or the Lagrange interpolation of $g$ in $\mathcal{S}_{\partial \Omega}^{h}$ in case $g$ being smooth enough (see [23. for more details). 
Definition 3.3. (i) For any fixed $j \in H_{-c_{f}}^{-1 / 2}(\partial \Omega)$ the operator $\mathcal{N}^{h}: \mathcal{Q} \rightarrow \mathcal{V}_{1, \diamond}^{h}$ mapping each $q \in \mathcal{Q}$ to the unique solution $u^{h}=: \mathcal{N}_{q}^{h} j$ of the variational equation (3.6) is called the discrete Neumann operator.

(ii) For any fixed $g \in H_{\diamond}^{1 / 2}(\partial \Omega)$ the operator $\mathcal{D}^{h}: \mathcal{Q} \rightarrow \mathcal{V}_{1, \diamond}^{h}$ mapping each $q \in \mathcal{Q}$ to the unique solution $v^{h}=: \mathcal{D}_{q}^{h} g$ of the variational equation 3.8 is called the discrete Dirichlet operator.

Next, the discretization space for the sought conductivity is defined by

$$
\mathcal{Q}_{a d}^{h}:=\mathcal{Q} \cap \mathcal{V}_{1}^{h} \subset \mathcal{Q} \cap B V(\Omega)=\mathcal{Q}_{a d} .
$$

Then, using the discrete operators $\mathcal{N}^{h}$ and $\mathcal{D}^{h}$ in Definition 3.3 , we introduce the discrete cost functional

$$
\Upsilon_{\rho, \delta}^{h}(q):=\mathcal{J}_{\delta}^{h}(q)+\rho \int_{\Omega} \sqrt{|\nabla q|^{2}+\epsilon^{h}},
$$

where $q \in \mathcal{Q}_{a d}^{h}, \epsilon^{h}$ is a positive function of the mesh size $h$ satisfying $\lim _{h \rightarrow 0} \epsilon^{h}=0$ and

$$
\mathcal{J}_{\delta}^{h}(q):=\int_{\Omega} q \nabla\left(\mathcal{N}_{q}^{h} j_{\delta}-\mathcal{D}_{q}^{h} g_{\delta}\right) \cdot \nabla\left(\mathcal{N}_{q}^{h} j_{\delta}-\mathcal{D}_{q}^{h} g_{\delta}\right) \text { with } q \in \mathcal{Q} .
$$

The positive function $\epsilon^{h}$ above acts as a smoothing parameter for the total variation.

Theorem 3.4. For any fixed $h, \rho$ and $\delta$ the minimization problem

$$
\min _{q \in \mathcal{Q}_{a d}^{h}} \Upsilon_{\rho, \delta}^{h}(q)
$$

attains a solution $q_{\rho, \delta}^{h}$, which is called the discrete regularized solution of the identication problem.

Proof. We first note that $\mathcal{Q}_{a d}^{h}$ is a compact subset of the finite dimensional space $\mathcal{V}_{1}^{h}$. Let $\left(q_{n}\right) \subset \mathcal{Q}_{a d}^{h}$ be a minimizing sequence of the problem $\left(\mathcal{P}_{\rho, \delta}^{h}\right)$, i.e.,

$$
\lim _{n \rightarrow \infty} \Upsilon_{\rho, \delta}^{h}\left(q_{n}\right)=\inf _{q \in \mathcal{Q}_{a d}^{h}} \Upsilon_{\rho, \delta}^{h}(q) .
$$

Then a subsequence of $\left(q_{n}\right)$ which is denoted by the same symbol and an element $q \in \mathcal{Q}_{a d}^{h}$ exist such that $\left(q_{n}\right)$ converges to $q$ in the $H^{1}(\Omega)$-norm. We have that

$$
\begin{aligned}
& \left|\int_{\Omega} \sqrt{\left|\nabla q_{n}\right|^{2}+\epsilon^{h}}-\int_{\Omega} \sqrt{|\nabla q|^{2}+\epsilon^{h}}\right| \leq \int_{\Omega} \frac{\left.|| \nabla q_{n}\right|^{2}-|\nabla q|^{2} \mid}{\sqrt{\left|\nabla q_{n}\right|^{2}+\epsilon^{h}}+\sqrt{|\nabla q|^{2}+\epsilon^{h}}} \\
& \leq \frac{1}{2 \sqrt{\epsilon^{h}}} \int_{\Omega}\left|\nabla q_{n}-\nabla q\right|\left|\nabla q_{n}+\nabla q\right| \leq \frac{1}{2 \sqrt{\epsilon^{h}}}\left(\int_{\Omega}\left|\nabla q_{n}-\nabla q\right|^{2}\right)^{1 / 2}\left(\int_{\Omega}\left|\nabla q_{n}+\nabla q\right|^{2}\right)^{1 / 2} \\
& \leq \frac{1}{2 \sqrt{\epsilon^{h}}}\left\|q_{n}-q\right\|_{H^{1}(\Omega)}\left(\left\|q_{n}-q\right\|_{H^{1}(\Omega)}+2\|q\|_{H^{1}(\Omega)}\right) \rightarrow 0 \text { as } n \rightarrow \infty .
\end{aligned}
$$

On the other hand, similarly to Lemma 2.4 , we can prove that the sequence $\left(\mathcal{N}_{q_{n}}^{h} j_{\delta}, \mathcal{D}_{q_{n}}^{h} g_{\delta}\right)$ converges to $\left(\mathcal{N}_{q}^{h} j_{\delta}, \mathcal{D}_{q}^{h} g_{\delta}\right)$ in the $H^{1}(\Omega) \times H^{1}(\Omega)$-norm as $n$ goes to $\infty$ and then obtain

$$
\lim _{n \rightarrow \infty} \mathcal{J}_{\delta}^{h}\left(q_{n}\right)=\mathcal{J}_{\delta}^{h}(q)
$$

Thus, it follows from $3.14-(3.16)$ that

$$
\Upsilon_{\rho, \delta}^{h}(q)=\lim _{n \rightarrow \infty} \Upsilon_{\rho, \delta}^{h}\left(q_{n}\right)=\inf _{q \in \mathcal{Q}_{a d}^{h}} \Upsilon_{\rho, \delta}^{h}(q),
$$

which finishes the proof. 


\section{Convergence}

From now on $C$ is a generic positive constant which is independent of the mesh size $h$ of $\mathcal{T}^{h}$, the noise level $\delta$ and the regularization parameter $\rho$. The following result shows the stability of the finite element method for the regularized identification problem.

Theorem 4.1. Let $\left(h_{n}\right)_{n}$ be a sequence with $\lim _{n \rightarrow \infty} h_{n}=0$ and $\left(j_{\delta_{n}}, g_{\delta_{n}}\right)$ be a sequence in $H_{-c_{f}}^{-1 / 2}(\partial \Omega) \times$ $H_{\diamond}^{1 / 2}(\partial \Omega)$ converging to $\left(j_{\delta}, g_{\delta}\right)$ in the $H^{-1 / 2}(\partial \Omega) \times H^{1 / 2}(\partial \Omega)$-norm. For a fixed regularization parameter $\rho>0$ let $q_{\rho, \delta_{n}}^{h_{n}} \in \mathcal{Q}_{a d}^{h_{n}}$ be a minimizer of $\left(\mathcal{P}_{\rho, \delta_{n}}^{h_{n}}\right)$ for each $n \in \mathbb{N}$. Then a subsequence of $\left(q_{\rho, \delta_{n}}^{h_{n}}\right)$ not relabelled and an element $q_{\rho, \delta} \in \mathcal{Q}_{a d}$ exist such that

$$
\lim _{n \rightarrow \infty}\left\|q_{\rho, \delta_{n}}^{h_{n}}-q_{\rho, \delta}\right\|_{L^{1}(\Omega)}=0 \text { and } \lim _{n \rightarrow \infty} \int_{\Omega}\left|\nabla q_{\rho, \delta_{n}}^{h_{n}}\right|=\int_{\Omega}\left|\nabla q_{\rho, \delta}\right| .
$$

Furthermore, $q_{\rho, \delta}$ is a solution to $\left(\mathcal{P}_{\rho, \delta}\right)$.

To prove the theorem, we need the auxiliary results, starting with the following estimates.

Lemma 4.2. Let $\left(j_{1}, g_{1}\right)$ and $\left(j_{2}, g_{2}\right)$ be arbitrary in $H_{-c_{f}}^{-1 / 2}(\partial \Omega) \times H_{\diamond}^{1 / 2}(\partial \Omega)$. Then the estimates

$$
\left\|\mathcal{N}_{q}^{h} j_{1}-\mathcal{N}_{q}^{h} j_{2}\right\|_{H^{1}(\Omega)} \leq \frac{1+C_{\diamond}^{\Omega}}{C_{\diamond}^{\Omega} \underline{q}}\|\gamma\|_{\mathcal{L}\left(H^{1}(\Omega), H^{1 / 2}(\partial \Omega)\right)}\left\|j_{1}-j_{2}\right\|_{H^{-1 / 2}(\partial \Omega)}
$$

and

$$
\left\|\mathcal{D}_{q}^{h} g_{1}-\mathcal{D}_{q}^{h} g_{2}\right\|_{H^{1}(\Omega)} \leq\left(\frac{1+C_{\diamond}^{\Omega}}{C_{\diamond}^{\Omega} \underline{q}} \bar{q}+1\right)\left\|\Pi_{\diamond}^{h}\right\|_{\mathcal{L}\left(H^{1}(\Omega), H^{1}(\Omega)\right)}\left\|\gamma^{-1}\right\|_{\mathcal{L}\left(H^{1 / 2}(\partial \Omega), H^{1}(\Omega)\right)}\left\|g_{1}-g_{2}\right\|_{H^{1 / 2}(\partial \Omega)}
$$

hold for all $q \in \mathcal{Q}$ and $h>0$.

Proof. According to the definition of the discrete Neumann operator, we have for all $\varphi^{h} \in \mathcal{V}_{1, \diamond}^{h}$ that

$$
\int_{\Omega} q \nabla \mathcal{N}_{q}^{h} j_{i} \cdot \nabla \varphi^{h}=\left\langle j_{i}, \gamma \varphi^{h}\right\rangle+\left(f, \varphi^{h}\right) \text { with } i=1,2
$$

Thus, $\Phi_{\mathcal{N}}^{h}:=\mathcal{N}_{q}^{h} j_{1}-\mathcal{N}_{q}^{h} j_{2}$ is the unique solution to the variational problem

$$
\int_{\Omega} q \nabla \Phi_{\mathcal{N}}^{h} \cdot \nabla \varphi^{h}=\left\langle j_{1}-j_{2}, \gamma \varphi^{h}\right\rangle
$$

for all $\varphi^{h} \in \mathcal{V}_{1, \diamond}^{h}$ and so that 4.1) follows. Similarly, we also obtain 4.2), which finishes the proof.

Lemma 4.3. Let $\left(h_{n}\right)_{n}$ be a sequence with $\lim _{n \rightarrow \infty} h_{n}=0$ and $\left(j_{\delta_{n}}, g_{\delta_{n}}\right) \subset H_{-c_{f}}^{-1 / 2}(\partial \Omega) \times H_{\diamond}^{1 / 2}(\partial \Omega)$ be a sequence converging to $\left(j_{\delta}, g_{\delta}\right)$ in the $H^{-1 / 2}(\partial \Omega) \times H^{1 / 2}(\partial \Omega)$-norm. Then for any fixed $q \in \mathcal{Q}$ the limit

$$
\lim _{n \rightarrow \infty} \mathcal{J}_{\delta_{n}}^{h_{n}}(q)=\mathcal{J}_{\delta}(q)
$$

holds. Furthermore, if $\left(q_{n}\right)$ is a sequence in $\mathcal{Q}$ which converges to $q$ in the $L^{1}(\Omega)$-norm, then the sequence $\left(\mathcal{N}_{q_{n}}^{h_{n}} j_{\delta_{n}}, \mathcal{D}_{q_{n}}^{h_{n}} g_{\delta_{n}}\right)$ converges to $\left(\mathcal{N}_{q} j_{\delta}, \mathcal{D}_{q} g_{\delta}\right)$ in the $H^{1}(\Omega) \times H^{1}(\Omega)$-norm and the limit

$$
\lim _{n \rightarrow \infty} \mathcal{J}_{\delta_{n}}^{h_{n}}\left(q_{n}\right)=\mathcal{J}_{\delta}(q)
$$

also holds. 
Proof. We get for any fixed $q \in \mathcal{Q}$ that

$$
\mathcal{N}_{q}^{h_{n}} j_{\delta_{n}}-\mathcal{D}_{q}^{h_{n}} g_{\delta_{n}}=\left(\mathcal{N}_{q} j_{\delta}-\mathcal{D}_{q} g_{\delta}\right)+\left(\mathcal{N}_{q}^{h_{n}} j_{\delta_{n}}-\mathcal{N}_{q} j_{\delta}+\mathcal{D}_{q} g_{\delta}-\mathcal{D}_{q}^{h_{n}} g_{\delta_{n}}\right) .
$$

Thus, with $\Phi_{n}:=\mathcal{N}_{q}^{h_{n}} j_{\delta_{n}}-\mathcal{N}_{q} j_{\delta}+\mathcal{D}_{q} g_{\delta}-\mathcal{D}_{q}^{h_{n}} g_{\delta_{n}}$ we have

$$
\begin{aligned}
\mathcal{J}_{\delta_{n}}^{h_{n}}(q) & =\int_{\Omega} q \nabla\left(\mathcal{N}_{q}^{h_{n}} j_{\delta_{n}}-\mathcal{D}_{q}^{h_{n}} g_{\delta_{n}}\right) \cdot \nabla\left(\mathcal{N}_{q}^{h_{n}} j_{\delta_{n}}-\mathcal{D}_{q}^{h_{n}} g_{\delta_{n}}\right) \\
& =\mathcal{J}_{\delta}(q)+\int_{\Omega} q \nabla \Phi_{n} \cdot \nabla \Phi_{n}+2 \int_{\Omega} q \nabla\left(\mathcal{N}_{q} j_{\delta}-\mathcal{D}_{q} g_{\delta}\right) \cdot \nabla \Phi_{n} .
\end{aligned}
$$

Applying Lemma 4.2, we infer that

$$
\begin{aligned}
\left\|\mathcal{N}_{q}^{h_{n}} j_{\delta_{n}}-\mathcal{N}_{q} j_{\delta}\right\|_{H^{1}(\Omega)} & \leq\left\|\mathcal{N}_{q}^{h_{n}} j_{\delta}-\mathcal{N}_{q} j_{\delta}\right\|_{H^{1}(\Omega)}+\left\|\mathcal{N}_{q}^{h_{n}} j_{\delta_{n}}-\mathcal{N}_{q}^{h_{n}} j_{\delta}\right\|_{H^{1}(\Omega)} \\
& \leq\left\|\mathcal{N}_{q}^{h_{n}} j_{\delta}-\mathcal{N}_{q} j_{\delta}\right\|_{H^{1}(\Omega)}+C\left\|j_{\delta_{n}}-j_{\delta}\right\|_{H^{-1 / 2}(\partial \Omega)} \rightarrow 0 \text { as } n \rightarrow \infty,
\end{aligned}
$$

where we used the limit

$$
\lim _{n \rightarrow \infty}\left\|\mathcal{N}_{q}^{h_{n}} j_{\delta}-\mathcal{N}_{q} j_{\delta}\right\|_{H^{1}(\Omega)}=0
$$

due to the standard theory (see, for example, [11, 18]). Similarly, we also have

$$
\left\|\mathcal{D}_{q}^{h_{n}} g_{\delta_{n}}-\mathcal{D}_{q} g_{\delta}\right\|_{H^{1}(\Omega)} \rightarrow 0 \text { as } n \rightarrow \infty .
$$

We thus get that

$$
\left\|\Phi_{n}\right\|_{H^{1}(\Omega)} \leq\left\|\mathcal{N}_{q}^{h_{n}} j_{\delta_{n}}-\mathcal{N}_{q} j_{\delta}\right\|_{H^{1}(\Omega)}+\left\|\mathcal{D}_{q} g_{\delta}-\mathcal{D}_{q}^{h_{n}} g_{\delta_{n}}\right\|_{H^{1}(\Omega)} \rightarrow 0 \text { as } n \rightarrow \infty .
$$

Therefore, we obtain that

$$
\lim _{n \rightarrow \infty}\left|\int_{\Omega} q \nabla \Phi_{n} \cdot \nabla \Phi_{n}+2 \int_{\Omega} q \nabla\left(\mathcal{N}_{q} j_{\delta}-\mathcal{D}_{q} g_{\delta}\right) \cdot \nabla \Phi_{n}\right| \leq C \lim _{n \rightarrow \infty}\left(\left\|\Phi_{n}\right\|_{H^{1}(\Omega)}^{2}+\left\|\Phi_{n}\right\|_{H^{1}(\Omega)}\right)=0
$$

and 4.3 then follows.

Next, for $q_{n}$ converging to $q$ in $L^{1}(\Omega)$, hence, along a subsequence again denoted by $\left(q_{n}\right)_{n}$, pointwise almost everywhere, by (3.6) and (2.4), we have

$$
\begin{aligned}
\int_{\Omega} q_{n} \nabla \mathcal{N}_{q_{n}}^{h_{n}} j_{\delta_{n}} \cdot \nabla \varphi^{h_{n}} & =\left\langle j_{\delta_{n}}, \gamma \varphi^{h_{n}}\right\rangle+\left(f, \varphi^{h_{n}}\right)=\left\langle j_{\delta}, \gamma \varphi^{h_{n}}\right\rangle+\left(f, \varphi^{h_{n}}\right)+\left\langle j_{\delta_{n}}-j_{\delta}, \gamma \varphi^{h_{n}}\right\rangle \\
& =\int_{\Omega} q \nabla \mathcal{N}_{q} j_{\delta} \cdot \nabla \varphi^{h_{n}}+\left\langle j_{\delta_{n}}-j_{\delta}, \gamma \varphi^{h_{n}}\right\rangle
\end{aligned}
$$

for all $\varphi^{h_{n}} \in \mathcal{V}_{1, \diamond}^{h_{n}}$ which implies that

$$
\begin{aligned}
\int_{\Omega} q_{n} \nabla & \left(\mathcal{N}_{q_{n}}^{h_{n}} j_{\delta_{n}}-\Pi_{\diamond}^{h_{n}} \mathcal{N}_{q} j_{\delta}\right) \cdot \nabla \varphi^{h_{n}} \\
& =\int_{\Omega} q \nabla \mathcal{N}_{q} j_{\delta} \cdot \nabla \varphi^{h_{n}}-\int_{\Omega} q_{n} \nabla \Pi_{\diamond}^{h_{n}} \mathcal{N}_{q} j_{\delta} \cdot \nabla \varphi^{h_{n}}+\left\langle j_{\delta_{n}}-j_{\delta}, \gamma \varphi^{h_{n}}\right\rangle \\
& =\int_{\Omega}\left(q-q_{n}\right) \nabla \mathcal{N}_{q} j_{\delta} \cdot \nabla \varphi^{h_{n}}+\int_{\Omega} q_{n} \nabla\left(\mathcal{N}_{q} j_{\delta}-\Pi_{\diamond}^{h_{n}} \mathcal{N}_{q} j_{\delta}\right) \cdot \nabla \varphi^{h_{n}}+\left\langle j_{\delta_{n}}-j_{\delta}, \gamma \varphi^{h_{n}}\right\rangle,
\end{aligned}
$$

where the operator $\Pi_{\diamond}^{h_{n}}$ is defined according to Lemma 3.1. Taking $\varphi^{h_{n}}=\mathcal{N}_{q_{n}}^{h_{n}} j_{\delta_{n}}-\Pi_{\diamond}^{h_{n}} \mathcal{N}_{q} j_{\delta} \in \mathcal{V}_{1, \diamond}^{h}$, by 2.2 and using the Cauchy-Schwarz inequality, we get

$$
\begin{aligned}
\frac{C_{\diamond}^{\Omega} \underline{q}}{1+C_{\diamond}^{\Omega}} \| & \mathcal{N}_{q_{n}}^{h_{n}} j_{\delta_{n}}-\Pi_{\diamond}^{h_{n}} \mathcal{N}_{q} j_{\delta} \|_{H^{1}(\Omega)}^{2} \\
\leq & \left(\int_{\Omega}\left|q-q_{n}\right|^{2}\left|\nabla \mathcal{N}_{q} j_{\delta}\right|^{2}\right)^{1 / 2}\left\|\mathcal{N}_{q_{n}}^{h_{n}} j_{\delta_{n}}-\Pi_{\diamond}^{h_{n}} \mathcal{N}_{q} j_{\delta}\right\|_{H^{1}(\Omega)} \\
& +\bar{q}\left\|\mathcal{N}_{q} j_{\delta}-\Pi_{\diamond}^{h_{n}} \mathcal{N}_{q} j_{\delta}\right\|_{H^{1}(\Omega)}\left\|\mathcal{N}_{q_{n}}^{h_{n}} j_{\delta_{n}}-\Pi_{\diamond}^{h_{n}} \mathcal{N}_{q} j_{\delta}\right\|_{H^{1}(\Omega)} \\
& +\|\gamma\|_{\mathcal{L}\left(H^{1}(\Omega), H^{1 / 2}(\partial \Omega)\right)}\left\|j_{\delta_{n}}-j_{\delta}\right\|_{H^{-1 / 2}(\partial \Omega)}\left\|\mathcal{N}_{q_{n}}^{h_{n}} j_{\delta_{n}}-\Pi_{\diamond}^{h_{n}} \mathcal{N}_{q} j_{\delta}\right\|_{H^{1}(\Omega)}
\end{aligned}
$$


and so that

$$
\begin{aligned}
\frac{C_{\diamond}^{\Omega} \underline{q}}{1+C_{\diamond}^{\Omega}}\left\|\mathcal{N}_{q_{n}}^{h_{n}} j_{\delta_{n}}-\Pi_{\diamond}^{h_{n}} \mathcal{N}_{q} j_{\delta}\right\|_{H^{1}(\Omega)} \leq & \left(\int_{\Omega}\left|q-q_{n}\right|^{2}\left|\nabla \mathcal{N}_{q} j_{\delta}\right|^{2}\right)^{1 / 2}+\bar{q}\left\|\mathcal{N}_{q} j_{\delta}-\Pi_{\diamond}^{h_{n}} \mathcal{N}_{q} j_{\delta}\right\|_{H^{1}(\Omega)} \\
& +\|\gamma\|_{\mathcal{L}\left(H^{1}(\Omega), H^{1 / 2}(\partial \Omega)\right)}\left\|j_{\delta_{n}}-j_{\delta}\right\|_{H^{-1 / 2}(\partial \Omega)} \rightarrow 0 \text { as } n \rightarrow \infty,
\end{aligned}
$$

by the Lebesgue dominated convergence theorem and (3.1). Thus, we infer from the triangle inequality that

$$
\left\|\mathcal{N}_{q_{n}}^{h_{n}} j_{\delta_{n}}-\mathcal{N}_{q} j_{\delta}\right\|_{H^{1}(\Omega)} \leq\left\|\mathcal{N}_{q_{n}}^{h_{n}} j_{\delta_{n}}-\Pi_{\diamond}^{h_{n}} \mathcal{N}_{q} j_{\delta}\right\|_{H^{1}(\Omega)}+\left\|\Pi_{\diamond}^{h_{n}} \mathcal{N}_{q} j_{\delta}-\mathcal{N}_{q} j_{\delta}\right\|_{H^{1}(\Omega)} \rightarrow 0 \text { as } n \rightarrow \infty
$$

Similarly, using 2.8 and 3.8, for all $\psi^{h_{n}} \in \mathcal{V}_{1,0}^{h_{n}}$ we arrive at

$$
\int_{\Omega} q_{n} \nabla\left(\mathcal{D}_{q_{n}}^{h_{n}} g_{\delta}-\Pi_{\diamond}^{h_{n}} \mathcal{D}_{q} g_{\delta}\right) \cdot \nabla \psi^{h_{n}}=\int_{\Omega}\left(q-q_{n}\right) \nabla \mathcal{D}_{q} g_{\delta} \cdot \nabla \psi^{h_{n}}+\int_{\Omega} q_{n} \nabla\left(\mathcal{D}_{q} g_{\delta}-\Pi_{\diamond}^{h_{n}} \mathcal{D}_{q} g_{\delta}\right) \cdot \nabla \psi^{h_{n}} .
$$

We have

$$
\gamma \mathcal{D}_{q_{n}}^{h_{n}} g_{\delta}=\gamma\left(\Pi_{\diamond}^{h_{n}}\left(\gamma^{-1} g_{\delta}\right)\right)
$$

by Proposition 3.2 (ii). On the other hand, in view of 2.9 , we get $\mathcal{D}_{q} g_{\delta}=v_{0}+\gamma^{-1} g_{\delta}$ with $v_{0} \in H_{0}^{1}(\Omega)$, and therefore

$$
\gamma\left(\Pi_{\diamond}^{h_{n}} \mathcal{D}_{q} g_{\delta}\right)=\gamma\left(\Pi_{\diamond}^{h_{n}}\left(v_{0}+\gamma^{-1} g_{\delta}\right)\right)=\gamma\left(\Pi_{\diamond}^{h_{n}} v_{0}\right)+\gamma\left(\Pi_{\diamond}^{h_{n}}\left(\gamma^{-1} g_{\delta}\right)\right)=\gamma\left(\Pi_{\diamond}^{h_{n}}\left(\gamma^{-1} g_{\delta}\right)\right),
$$

since $\gamma\left(\Pi_{\diamond}^{h_{n}} v_{0}\right)=0$. It follows from $4.7-4.8$ that

$$
\psi_{*}^{h_{n}}:=\mathcal{D}_{q_{n}}^{h_{n}} g_{\delta}-\Pi_{\diamond}^{h_{n}} \mathcal{D}_{q} g_{\delta} \in \mathcal{V}_{1,0}^{h_{n}}
$$

Taking $\psi^{h_{n}}:=\psi_{*}^{h_{n}}$ in the above equation (4.6), it is deduced that

$$
\lim _{n \rightarrow \infty}\left\|\mathcal{D}_{q_{n}}^{h_{n}} g_{\delta}-\Pi_{\diamond}^{h_{n}} \mathcal{D}_{q} g_{\delta}\right\|_{H^{1}(\Omega)}=0
$$

Using Lemma 4.2, we therefore obtain that

$$
\begin{aligned}
\| \mathcal{D}_{q_{n}}^{h_{n}} g_{\delta_{n}} & -\mathcal{D}_{q} g_{\delta} \|_{H^{1}(\Omega)} \\
& \leq\left\|\mathcal{D}_{q_{n}}^{h_{n}} g_{\delta_{n}}-\mathcal{D}_{q_{n}}^{h_{n}} g_{\delta}\right\|_{H^{1}(\Omega)}+\left\|\mathcal{D}_{q_{n}}^{h_{n}} g_{\delta}-\Pi_{\diamond}^{h_{n}} \mathcal{D}_{q} g_{\delta}\right\|_{H^{1}(\Omega)}+\left\|\Pi_{\diamond}^{h_{n}} \mathcal{D}_{q} g_{\delta}-\mathcal{D}_{q} g_{\delta}\right\|_{H^{1}(\Omega)} \\
& \leq C\left\|g_{\delta_{n}}-g_{\delta}\right\|_{H^{1 / 2}(\partial \Omega)}+\left\|\mathcal{D}_{q_{n}}^{h_{n}} g_{\delta}-\Pi_{\diamond}^{h_{n}} \mathcal{D}_{q} g_{\delta}\right\|_{H^{1}(\Omega)}+\left\|\Pi_{\diamond}^{h_{n}} \mathcal{D}_{q} g_{\delta}-\mathcal{D}_{q} g_{\delta}\right\|_{H^{1}(\Omega)} \\
& \rightarrow 0 \text { as } n \rightarrow \infty .
\end{aligned}
$$

Since $\left(q_{n}\right)$ converges to $q$ in the $L^{1}(\Omega)$-norm while the sequence $\left(\mathcal{N}_{q_{n}}^{h_{n}} j_{\delta_{n}}, \mathcal{D}_{q_{n}}^{h_{n}} g_{\delta_{n}}\right)$ converges to $\left(\mathcal{N}_{q} j_{\delta}, \mathcal{D}_{q} g_{\delta}\right)$ in the $H^{1}(\Omega) \times H^{1}(\Omega)$-norm, we conclude, similarly to the proof of Lemma 2.4 that

$$
\lim _{n \rightarrow \infty} \mathcal{J}_{\delta_{n}}^{h_{n}}\left(q_{n}\right)=\mathcal{J}_{\delta}(q),
$$

which finishes the proof.

Proof of Theorem 4.1. To simplify notation we write $q_{n}:=q_{\rho, \delta_{n}}^{h_{n}}$. Let $q \in \mathcal{Q}_{a d}$ be arbitrary. Using Lemma 2.6. for any fixed $\alpha \in(0,1)$ an element $q^{\alpha} \in C^{\infty}(\Omega)$ exists such that

$$
\left\|q-q^{\alpha}\right\|_{L^{1}(\Omega)} \leq \bar{C} \alpha \text { and } \int_{\Omega}\left|\nabla q^{\alpha}\right| \leq \bar{C} \alpha+\int_{\Omega}|\nabla q|,
$$

where the positive constant $\bar{C}$ is independent of $\alpha$. Setting

$$
q_{P}^{\alpha}:=\max \left(\underline{q}, \min \left(q^{\alpha}, \bar{q}\right)\right) \in W^{1, \infty}(\Omega) \cap \mathcal{Q} \subset \mathcal{Q}_{a d} \text { and } q_{n}^{\alpha}:=I_{1}^{h_{n}} q_{P}^{\alpha} \in \mathcal{Q}_{a d}^{h_{n}},
$$


where

$$
I_{1}^{h}: W^{1, p}(\Omega) \hookrightarrow C(\bar{\Omega}) \rightarrow \mathcal{V}_{1}^{h} \text { with } p>d
$$

is the usual nodal value interpolation operator. Since the sequence $\left(q_{n}^{\alpha}\right)$ converges to $q_{P}^{\alpha}$ in the $H^{1}(\Omega)$-norm as $n$ tends to $\infty$ (see, for example, [11, 18]), we get the equation

$$
\lim _{n \rightarrow \infty} \int_{\Omega} \sqrt{\left|\nabla q_{n}^{\alpha}\right|^{2}+\epsilon^{h_{n}}}=\int_{\Omega}\left|\nabla q_{P}^{\alpha}\right|
$$

Indeed, we have that

$$
\left|\int_{\Omega} \sqrt{\left|\nabla q_{n}^{\alpha}\right|^{2}+\epsilon^{h_{n}}}-\int_{\Omega}\right| \nabla q_{n}^{\alpha}|| \leq \int_{\Omega} \frac{\epsilon^{h_{n}}}{\sqrt{\left|\nabla q_{n}^{\alpha}\right|^{2}+\epsilon^{h_{n}}}+\left|\nabla q_{n}^{\alpha}\right|} \leq|\Omega| \sqrt{\epsilon^{h_{n}}} \rightarrow 0 \text { as } n \rightarrow \infty
$$

and by the reverse triangle as well as the Cauchy Schwarz inequality

$$
\left|\int_{\Omega}\right| \nabla q_{n}^{\alpha}\left|-\int_{\Omega}\right| \nabla q_{P}^{\alpha}|| \leq\left\|\nabla q_{n}^{\alpha}-\nabla q_{P}^{\alpha}\right\|_{L^{1}(\Omega)} \leq|\Omega|^{1 / 2}\left\|\nabla q_{n}^{\alpha}-\nabla q_{P}^{\alpha}\right\|_{L^{2}(\Omega)} \rightarrow 0 \text { as } n \rightarrow \infty
$$

so that (4.10) follows from the triangle inequality. By 4.9 ) and the fact that $q_{P}^{\alpha}$ is constant on $\{x \in$ $\left.\Omega \mid q_{P}^{\alpha}(x) \neq q^{\alpha}(x)\right\}$, we have that

$$
\int_{\Omega}\left|\nabla q_{P}^{\alpha}\right|=\int_{\left\{x \in \Omega \mid q_{P}^{\alpha}(x)=q^{\alpha}(x)\right\}}\left|\nabla q_{P}^{\alpha}\right| \leq \int_{\Omega}\left|\nabla q^{\alpha}\right| \leq \bar{C} \alpha+\int_{\Omega}|\nabla q| .
$$

By the optimality of $q_{n}$, we get for all $n \in \mathbb{N}$ that

$$
\mathcal{J}_{\delta_{n}}^{h_{n}}\left(q_{n}\right)+\rho \int_{\Omega} \sqrt{\left|\nabla q_{n}\right|^{2}+\epsilon^{h_{n}}} \leq \mathcal{J}_{\delta_{n}}^{h_{n}}\left(q_{n}^{\alpha}\right)+\rho \int_{\Omega} \sqrt{\left|\nabla q_{n}^{\alpha}\right|^{2}+\epsilon^{h_{n}}},
$$

where, by (3.7) and (3.9),

$$
\mathcal{J}_{\delta_{n}}^{h_{n}}\left(q_{n}^{\alpha}\right) \leq C
$$

holds for some $C$ independent of $n$ and $\alpha$. We then deduce from 4.10 4.12 that

$$
\int_{\Omega}\left|\nabla q_{n}\right| \leq \int_{\Omega} \sqrt{\left|\nabla q_{n}\right|^{2}+\epsilon^{h_{n}}} \leq C(\rho)
$$

for another constant $C(\rho)$ independent of $n$ and $\alpha$, but depending on $\rho$, so the sequence $\left(q_{n}\right)$ is bounded in the $B V(\Omega)$-norm. Thus, by Lemma 2.5 , a subsequence which is denoted by the same symbol and an element $\widehat{q} \in \mathcal{Q}_{a d}$ exist such that $\left(q_{n}\right)$ converges to $\widehat{q}$ in the $L^{1}(\Omega)$-norm and

$$
\int_{\Omega}|\nabla \widehat{q}| \leq \liminf _{n \rightarrow \infty} \int_{\Omega}\left|\nabla q_{n}\right| \leq \liminf _{n \rightarrow \infty} \int_{\Omega} \sqrt{\left|\nabla q_{n}\right|^{2}+\epsilon^{h_{n}}} .
$$

Furthermore, due to Lemma 4.3 we get that

$$
\mathcal{J}_{\delta}(\widehat{q})=\lim _{n \rightarrow \infty} \mathcal{J}_{\delta_{n}}^{h_{n}}\left(q_{n}\right)
$$

and

$$
\mathcal{J}_{\delta}\left(q_{P}^{\alpha}\right)=\lim _{n \rightarrow \infty} \mathcal{J}_{\delta_{n}}^{h_{n}}\left(q_{n}^{\alpha}\right) .
$$

Therefore, by $4.10-4.15$, we have that

$$
\begin{aligned}
\mathcal{J}_{\delta}(\widehat{q})+\rho \int_{\Omega}|\nabla \widehat{q}| & \leq \lim _{n \rightarrow \infty} \mathcal{J}_{\delta_{n}}^{h_{n}}\left(q_{n}\right)+\liminf _{n \rightarrow \infty} \rho \int_{\Omega} \sqrt{\left|\nabla q_{n}\right|^{2}+\epsilon^{h_{n}}} \text {, by } 4.14 \text { and } \\
& =\liminf _{n \rightarrow \infty}\left(\mathcal{J}_{\delta_{n}}^{h_{n}}\left(q_{n}\right)+\rho \int_{\Omega} \sqrt{\left|\nabla q_{n}\right|^{2}+\epsilon^{h_{n}}}\right) \\
& \leq \liminf _{n \rightarrow \infty}\left(\mathcal{J}_{\delta_{n}}^{h_{n}}\left(q_{n}^{\alpha}\right)+\rho \int_{\Omega} \sqrt{\left|\nabla q_{n}^{\alpha}\right|^{2}+\epsilon^{h_{n}}}\right), \text { by } 4.12 \\
& =\mathcal{J}_{\delta}\left(q_{P}^{\alpha}\right)+\rho \int_{\Omega}\left|\nabla q_{P}^{\alpha}\right|, \text { by } 4.15 \text { and } 4.10 \\
& \leq \mathcal{J}_{\delta}\left(q_{P}^{\alpha}\right)+\rho \int_{\Omega}|\nabla q|+\bar{C} \alpha \rho, \text { by 4.11. }
\end{aligned}
$$


Now, by the definition of $q_{P}^{\alpha}$, we get $\left|q_{P}^{\alpha}-q\right| \leq\left|q^{\alpha}-q\right|$ a.e. in $\Omega$ and therefore

$$
\left\|q_{P}^{\alpha}-q\right\|_{L^{1}(\Omega)} \leq\left\|q^{\alpha}-q\right\|_{L^{1}(\Omega)} \leq \bar{C} \alpha .
$$

Sending $\alpha$ to zero in the last inequality and applying Lemma 2.4, we arrive at

$$
\mathcal{J}_{\delta}(\widehat{q})+\rho \int_{\Omega}|\nabla \widehat{q}| \leq \mathcal{J}_{\delta}(q)+\rho \int_{\Omega}|\nabla q|,
$$

where $\widehat{q} \in \mathcal{Q}_{a d}$ and $q \in \mathcal{Q}_{a d}$ is arbitrary. This means that $\widehat{q}$ is a solution to $\left(\mathcal{P}_{\rho, \delta}\right)$ and $\left(q_{n}\right)$ converges to $\widehat{q}$ in the $L^{1}(\Omega)$-norm.

Next, as above, from $\widehat{q}$ we can obtain $\widehat{q}^{\alpha}, \widehat{q}_{P}^{\alpha}, \widehat{q}_{n}^{\alpha}$ and note that $\left(\widehat{q}_{n}^{\alpha}\right)$ converges to $\widehat{q}_{P}^{\alpha}$ in the $H^{1}(\Omega)$-norm, so also in the $L^{1}(\Omega)$-norm, as $n$ tends to $\infty$ while $\left(\widehat{q}_{P}^{\alpha}\right)$ converges to $\widehat{q}$ in the $L^{1}(\Omega)$-norm as $\alpha$ tends to 0 . Then, by the optimality of $q_{n}$, we have that

$$
\mathcal{J}_{\delta_{n}}^{h_{n}}\left(q_{n}\right)+\rho \int_{\Omega} \sqrt{\left|\nabla q_{n}\right|^{2}+\epsilon^{h_{n}}} \leq \mathcal{J}_{\delta_{n}}^{h_{n}}\left(\widehat{q}_{n}^{\alpha}\right)+\rho \int_{\Omega} \sqrt{\left|\nabla \widehat{q}_{n}^{\alpha}\right|^{2}+\epsilon^{h_{n}}} .
$$

By 4.14, we then obtain that

$$
\begin{aligned}
\rho \limsup _{n \rightarrow \infty} \int_{\Omega}\left|\nabla q_{n}\right| & =\lim _{n \rightarrow \infty} \mathcal{J}_{\delta_{n}}^{h_{n}}\left(q_{n}\right)+\rho \limsup _{n \rightarrow \infty} \int_{\Omega}\left|\nabla q_{n}\right|-\mathcal{J}_{\delta}(\widehat{q}) \\
& \leq \limsup _{n \rightarrow \infty}\left(\mathcal{J}_{\delta_{n}}^{h_{n}}\left(q_{n}\right)+\rho \int_{\Omega} \sqrt{\left|\nabla q_{n}\right|^{2}+\epsilon^{h_{n}}}\right)-\mathcal{J}_{\delta}(\widehat{q}) \\
& \left.\leq \limsup _{n \rightarrow \infty}\left(\mathcal{J}_{\delta_{n}}^{h_{n}}\left(\widehat{q}_{n}^{\alpha}\right)+\rho \int_{\Omega} \sqrt{\left|\nabla \widehat{q}_{n}^{\alpha}\right|^{2}+\epsilon^{h_{n}}}\right)-\mathcal{J}_{\delta}(\widehat{q}), \text { by } 4.17\right) \\
& =\mathcal{J}_{\delta}\left(\widehat{q}_{P}^{\alpha}\right)+\rho \int_{\Omega}\left|\nabla \widehat{q}_{P}^{\alpha}\right|-\mathcal{J}_{\delta}(\widehat{q}), \text { by Lemma 4.3 } \\
& \leq \mathcal{J}_{\delta}\left(\widehat{q}_{P}^{\alpha}\right)+\rho \int_{\Omega}|\nabla \widehat{q}|+\bar{C} \alpha \rho-\mathcal{J}_{\delta}(\widehat{q}) .
\end{aligned}
$$

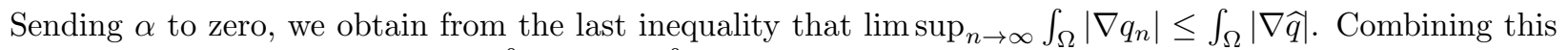
with 4.13, we conclude $\lim _{n \rightarrow \infty} \int_{\Omega}\left|\nabla q_{n}\right|=\int_{\Omega}|\nabla \widehat{q}|$, which finishes the proof.

Next we show convergence of the regularized finite element approximations to a solution of the identification problem. Before doing so, we introduce the notion of the total variation-minimizing solution.

Lemma 4.4. The problem

$$
\min _{q \in \mathcal{I}_{\mathcal{Q}_{a d}}\left(j^{\dagger}, g^{\dagger}\right)} \int_{\Omega}|\nabla q|
$$

attains a solution, which is called the total variation-minimizing solution of the identification problem, where

$$
\mathcal{I}_{\mathcal{Q}_{a d}}\left(j^{\dagger}, g^{\dagger}\right):=\left\{q \in \mathcal{Q}_{a d} \mid \Lambda_{q} j^{\dagger}=g^{\dagger}\right\}=\left\{q \in \mathcal{Q}_{a d} \mid \mathcal{N}_{q} j^{\dagger}=\mathcal{D}_{q} g^{\dagger}\right\} .
$$

Proof. By our assumption on consistency of the exact boundary data, the set $\mathcal{I}_{\mathcal{Q}_{a d}}\left(j^{\dagger}, g^{\dagger}\right)$ is non-empty. Let $\left(q_{n}\right) \subset \mathcal{I}_{\mathcal{Q}_{a d}}\left(j^{\dagger}, g^{\dagger}\right)$ be a minimizing sequence of the problem $(\mathcal{I} \mathcal{P})$, i.e.,

$$
\lim _{n \rightarrow \infty} \int_{\Omega}\left|\nabla q_{n}\right|=\inf _{q \in \mathcal{I}_{\mathcal{Q}_{a d}}\left(j^{\dagger}, g^{\dagger}\right)} \int_{\Omega}|\nabla q| .
$$

Then due to Lemma 2.5, a subsequence which is denoted by the same symbol and an element $\tilde{q} \in \mathcal{Q}_{a d}$ exist such that $\left(q_{n}\right)$ converges to $\tilde{q}$ in the $L^{1}(\Omega)$-norm and

$$
\int_{\Omega}|\nabla \tilde{q}| \leq \lim _{n \rightarrow \infty} \int_{\Omega}\left|\nabla q_{n}\right|
$$

On the other hand, by Lemma 2.4 we have that

$$
\left(\mathcal{N}_{q_{n}} j^{\dagger}, \mathcal{D}_{q_{n}} g^{\dagger}\right) \rightarrow\left(\mathcal{N}_{\tilde{q}} j^{\dagger}, \mathcal{D}_{\tilde{q}} g^{\dagger}\right) \text { in the } H^{1}(\Omega) \times H^{1}(\Omega) \text {-norm. }
$$


By the definition of the set $\mathcal{I}_{\mathcal{Q}_{a d}}\left(j^{\dagger}, g^{\dagger}\right)$, we get that $\mathcal{N}_{q_{n}} j^{\dagger}=\mathcal{D}_{q_{n}} g^{\dagger}$ which implies $\mathcal{N}_{\tilde{q}} j^{\dagger}=\mathcal{D}_{\tilde{q}} g^{\dagger}$. Combining this with (4.19) and (4.20), we conclude that

$$
\int_{\Omega}|\nabla \tilde{q}| \leq \inf _{q \in \mathcal{I}_{\mathcal{Q}_{a d}}\left(j^{\dagger}, g^{\dagger}\right)} \int_{\Omega}|\nabla q|,
$$

where $\tilde{q} \in \mathcal{I}_{\mathcal{Q}_{a d}}\left(j^{\dagger}, g^{\dagger}\right)$, which finishes the proof.

Remark 4.5. Note that due to the lack of strict convexity of the cost functional and the admissible set, a solution of $(\mathcal{I} \mathcal{P})$ may be nonunique.

Lemma 4.6. For any fixed $q \in \mathcal{Q}_{a d}$ an element $\widehat{q}^{h} \in \mathcal{Q}_{a d}^{h}$ exists such that

$$
\left\|\widehat{q}^{h}-q\right\|_{L^{1}(\Omega)} \leq C h|\log h|
$$

and

$$
\lim _{h \rightarrow 0} \int_{\Omega}\left|\nabla \widehat{q}^{h}\right|=\int_{\Omega}|\nabla q|
$$

In case $q \in W^{1, p}(\Omega) \hookrightarrow C(\bar{\Omega})$ with $p>d$ the above element $\widehat{q}^{h}$ can be taken as $I_{1}^{h} q$.

Proof. According to Lemma 2.6 for any fixed $\alpha \in(0,1)$ an element $q^{\alpha} \in C^{\infty}(\Omega)$ exists such that

$$
\left\|q-q^{\alpha}\right\|_{L^{1}(\Omega)} \leq \bar{C} \alpha, \int_{\Omega}\left|\nabla q^{\alpha}\right| \leq \bar{C} \alpha+\int_{\Omega}|\nabla q| \text { and } \int_{\Omega}\left|D^{2} q^{\alpha}\right| \leq \bar{C} \alpha^{-1} \int_{\Omega}|\nabla q|
$$

where the positive constant $\bar{C}$ is independent of $\alpha$. Setting

$$
q_{P}^{\alpha}:=\max \left(\underline{q}, \min \left(q^{\alpha}, \bar{q}\right)\right) \in W^{1, \infty}(\Omega) \cap \mathcal{Q} \subset \mathcal{Q}_{a d} \text { and } \widehat{q}^{h}:=I_{1}^{h} q_{P}^{\alpha} \in \mathcal{Q}_{a d}^{h},
$$

we then have

$$
\left|\widehat{q}^{h}(x)-q(x)\right|=\left|I_{1}^{h} q^{\alpha}(x)-q(x)\right| \text { a.e. in } \Omega_{1}:=\left\{x \in \Omega \mid \underline{q} \leq q^{\alpha} \leq \bar{q}\right\}
$$

and

$$
\left|\widehat{q}^{h}(x)-q(x)\right| \leq\left|q^{\alpha}(x)-q(x)\right| \text { a.e. in } \Omega \backslash \Omega_{1} .
$$

We thus have, using for example [11, Theorem 4.4.20], with an another positive constant $C$ independent of $\alpha$ that

$$
\begin{aligned}
\left\|\widehat{q}^{h}-q\right\|_{L^{1}(\Omega)} & \leq\left\|I_{1}^{h} q^{\alpha}-q\right\|_{L^{1}\left(\Omega_{1}\right)}+\left\|q^{\alpha}-q\right\|_{L^{1}\left(\Omega \backslash \Omega_{1}\right)} \\
& \leq\left\|I_{1}^{h} q^{\alpha}-q^{\alpha}\right\|_{L^{1}(\Omega)}+\left\|q-q^{\alpha}\right\|_{L^{1}\left(\Omega_{1}\right)}+\left\|q^{\alpha}-q\right\|_{L^{1}\left(\Omega \backslash \Omega_{1}\right)} \\
& \leq C h \int_{\Omega}\left|\nabla q^{\alpha}\right|+\left\|q-q^{\alpha}\right\|_{L^{1}(\Omega)} \\
& \leq C h\left(\bar{C} \alpha+\int_{\Omega}|\nabla q|\right)+\bar{C} \alpha \leq C(h+\alpha) \\
& \leq C h|\log h|
\end{aligned}
$$

for $\alpha \sim h|\log h|$. To establish the limit 4.22 we first note that

$$
\int_{\Omega}\left|\nabla I_{1}^{h} q_{P}^{\alpha}\right| \leq \int_{\Omega}\left|\nabla I_{1}^{h} q^{\alpha}\right|
$$

Indeed, we rewrite

$$
\int_{\Omega}\left|\nabla I_{1}^{h} q_{P}^{\alpha}\right|=\sum_{T \in \mathcal{T}_{1}^{h}} \int_{T}\left|\nabla I_{1}^{h} q_{P}^{\alpha}\right|+\sum_{T \in \mathcal{T}_{2}^{h}} \int_{T}\left|\nabla I_{1}^{h} q_{P}^{\alpha}\right|+\sum_{T \in \mathcal{T}^{h} \backslash\left(\mathcal{T}_{1}^{h} \cup \mathcal{T}_{2}^{h}\right)} \int_{T}\left|\nabla I_{1}^{h} q_{P}^{\alpha}\right|,
$$

where $\mathcal{T}_{1}^{h}$ includes all triangles $T \in \mathcal{T}^{h}$ with its vertices $x_{1}, \ldots, x_{d}, x_{d+1}$ at which

$$
\text { either } q^{\alpha}\left(x_{1}\right), \ldots, q^{\alpha}\left(x_{d+1}\right)<\underline{q} \text { or } q^{\alpha}\left(x_{1}\right), \ldots, q^{\alpha}\left(x_{d+1}\right)>\bar{q}
$$


while $\mathcal{T}_{2}^{h}$ consists all triangles $T \in \mathcal{T}^{h}$ with its vertices $x_{1}, \ldots, x_{d}, x_{d+1}$ at which

$$
q^{\alpha}\left(x_{1}\right), \ldots, q^{\alpha}\left(x_{d+1}\right) \in[\underline{q}, \bar{q}] .
$$

We then have that

$$
\sum_{T \in \mathcal{T}_{1}^{h}} \int_{T}\left|\nabla I_{1}^{h} q_{P}^{\alpha}\right|=0 \text { and } \sum_{T \in \mathcal{T}_{2}^{h}} \int_{T}\left|\nabla I_{1}^{h} q_{P}^{\alpha}\right|=\sum_{T \in \mathcal{T}_{2}^{h}} \int_{T}\left|\nabla I_{1}^{h} q^{\alpha}\right| .
$$

Now let $T \in \mathcal{T}^{h} \backslash\left(\mathcal{T}_{1}^{h} \cup \mathcal{T}_{2}^{h}\right)$ be arbitrary. In Cartesian coordinate system $O x z$ with $x \in \mathbb{R}^{d}$ we consider plane surfaces $z=I_{1}^{h} q_{P}^{\alpha}(x)$ and $z=I_{1}^{h} q^{\alpha}(x)$ with $x \in T$ and denote by $\vec{m}_{P}$ and $\vec{m}$ the constant unit normal on these surfaces in the upward $z$ direction, respectively. By the definition of the projection $q_{P}^{\alpha}$, we get $0<\left(\widehat{O z, \vec{m}_{P}}\right) \leq(\widehat{O z, \vec{m}})<\pi / 2$ and so that $0<\cos (\widehat{O z, \vec{m}}) \leq \cos \left(\widehat{O z, \vec{m}_{P}}\right)<1$. Since

$$
\cos (\widehat{O z, \vec{m}})=\frac{1}{\sqrt{\left|\nabla I_{1}^{h} q^{\alpha}\right|^{2}+1}} \text { and } \cos \left(\widehat{O z, \vec{m}_{P}}\right)=\frac{1}{\sqrt{\left|\nabla I_{1}^{h} q_{P}^{\alpha}\right|^{2}+1}},
$$

it follows that $\left|\nabla I_{1}^{h} q^{\alpha}(x)\right| \geq\left|\nabla I_{1}^{h} q_{P}^{\alpha}(x)\right|$ for all $x \in T$. We thus have that

$$
\sum_{T \in \mathcal{T}^{h} \backslash\left(\mathcal{T}_{1}^{h} \cup \mathcal{T}_{2}^{h}\right)} \int_{T}\left|\nabla I_{1}^{h} q_{P}^{\alpha}\right| \leq \sum_{T \in \mathcal{T}^{h} \backslash\left(\mathcal{T}_{1}^{h} \cup \mathcal{T}_{2}^{h}\right)} \int_{T}\left|\nabla I_{1}^{h} q^{\alpha}\right| .
$$

The inequality 4.23 is then directly deduced from (4.24) 4.26 . We therefore have with a constant $C$ independent of $\alpha$ that

$$
\begin{aligned}
\int_{\Omega}\left|\nabla \widehat{q}^{h}\right|-\int_{\Omega}|\nabla q| & =\int_{\Omega}\left|\nabla I_{1}^{h} q_{P}^{\alpha}\right|-\int_{\Omega}|\nabla q| \leq \int_{\Omega}\left|\nabla I_{1}^{h} q^{\alpha}\right|-\int_{\Omega}|\nabla q| \\
& \leq \int_{\Omega}\left|\nabla\left(I_{1}^{h} q^{\alpha}-q^{\alpha}\right)\right|+\int_{\Omega}\left|\nabla q^{\alpha}\right|-\int_{\Omega}|\nabla q| \\
& \leq C h \int_{\Omega}\left|D^{2} q^{\alpha}\right|+\bar{C} \alpha \\
& \leq C \bar{C} h \alpha^{-1} \int_{\Omega}|\nabla q|+\bar{C} \alpha \\
& \leq C\left(|\log h|^{-1}+h|\log h|\right) \rightarrow 0 \text { as } h \rightarrow 0 \text { and for } \alpha \sim h|\log h| .
\end{aligned}
$$

Combining this with 4.21 and Lemma 2.5, we obtain that

$$
\int_{\Omega}|\nabla q| \leq \liminf _{h \rightarrow 0} \int_{\Omega}\left|\nabla \widehat{q}^{h}\right| \leq \limsup _{h \rightarrow 0} \int_{\Omega}\left|\nabla \widehat{q}^{h}\right| \leq \int_{\Omega}|\nabla q|
$$

which finishes the proof.

Lemma 4.7. Let $(q, j, g) \in \mathcal{Q}_{a d} \times H_{-c_{f}}^{-1 / 2}(\partial \Omega) \times H_{\diamond}^{1 / 2}(\partial \Omega)$ be arbitrary. Then the convergence

$$
\widehat{\varrho}_{q}^{h}(j, g):=\left\|\mathcal{N}_{\widetilde{q}^{h}}^{h} j-\mathcal{N}_{q} j\right\|_{H^{1}(\Omega)}+\left\|\mathcal{D}_{\widetilde{q}^{h}}^{h} g-\mathcal{D}_{q} g\right\|_{H^{1}(\Omega)} \rightarrow 0 \text { as } h \rightarrow 0
$$

holds, where $\widehat{q}^{h}$ is generated from $q$ according to Lemma 4.6.

Proof. The assertion follows directly from Lemma 4.3 and Lemma 4.6 .

Additional smoothness assumptions enable an error estimate of $\widehat{\varrho}_{q}^{h}(j, g)$.

Lemma 4.8. Let $(q, j, g) \in \mathcal{Q}_{a d} \times H_{-c_{f}}^{-1 / 2}(\partial \Omega) \times H_{\diamond}^{1 / 2}(\partial \Omega)$ be arbitrary. Assume that $\mathcal{N}_{q} j, \mathcal{D}_{q} g \in H^{2}(\Omega)$. Then

$$
\widehat{\varrho}_{q}^{h}(j, g) \leq C_{r}(h|\log h|)^{r} \text { with } \begin{cases}r<1 / 2 & \text { if } d=2 \text { and } \\ r=1 / 3 & \text { if } d=3 .\end{cases}
$$


Proof. Due to Lemma 3.1, since $\mathcal{N}_{q} j \in H^{2}(\Omega)$, we get that

$$
\left\|\mathcal{N}_{q} j-\Pi_{\diamond}^{h} \mathcal{N}_{q} j\right\|_{H^{1}(\Omega)} \leq C h .
$$

Furthermore, it follows from Lemma 4.6 that

$$
\left\|q-\widehat{q}^{h}\right\|_{L^{p}(\Omega)}=\left(\int_{\Omega}\left|q-\widehat{q}^{h}\right|\left|q-\widehat{q}^{h}\right|^{p-1}\right)^{1 / p} \leq\left((2 \bar{q})^{p-1} C h|\log h|\right)^{1 / p} \leq C(h|\log h|)^{1 / p}
$$

for $p \in[1, \infty)$. Like in 4.5 , using $(3.6)$ and 2.4 , we infer that

$$
\int_{\Omega} \widehat{q}^{h} \nabla \mathcal{N}_{\widehat{q}^{h}}^{h} j \cdot \nabla \varphi^{h}=\left\langle j, \gamma \varphi^{h}\right\rangle+\left(f, \varphi^{h}\right)=\int_{\Omega} q \nabla \mathcal{N}_{q} j \cdot \nabla \varphi^{h}
$$

for all $\varphi^{h} \in \mathcal{V}_{1, \diamond}^{h}$ and obtain that

$$
\int_{\Omega} \widehat{q}^{h} \nabla\left(\mathcal{N}_{\widehat{q}^{h}}^{h} j-\Pi_{\diamond}^{h} \mathcal{N}_{q} j\right) \cdot \nabla \varphi^{h}=\int_{\Omega}\left(q-\widehat{q}^{h}\right) \nabla \mathcal{N}_{q} j \cdot \nabla \varphi^{h}+\int_{\Omega} \widehat{q}^{h} \nabla\left(\mathcal{N}_{q} j-\Pi_{\diamond}^{h} \mathcal{N}_{q} j\right) \cdot \nabla \varphi^{h} .
$$

Since $H^{2}(\Omega)$ is embedded in $W^{1, s}(\Omega)$ with

$$
s \begin{cases}<\infty & \text { if } d=2 \\ =6 & \text { if } d=3\end{cases}
$$

(see, for example, 1, Theorem 5.4]), it follows from Cauchy-Schwarz and Hölder's inequality that

$$
\begin{aligned}
\int_{\Omega}\left(q-\widehat{q}^{h}\right) \nabla \mathcal{N}_{q} j \cdot \nabla \varphi^{h} & \leq\left(\int_{\Omega}\left(q-\widehat{q}^{h}\right)^{2}\left|\nabla \mathcal{N}_{q} j\right|^{2}\right)^{1 / 2}\left(\int_{\Omega}\left|\nabla \varphi^{h}\right|^{2}\right)^{1 / 2} \\
& \leq\left\|q-\widehat{q}^{h}\right\|_{L^{2 s /(s-2)}(\Omega)}\left\|\nabla \mathcal{N}_{q} j\right\|_{L^{s}(\Omega)}\left\|\varphi^{h}\right\|_{H^{1}(\Omega)} \\
& \leq C\left\|q-\widehat{q}^{h}\right\|_{L^{2 s /(s-2)}(\Omega)}\left\|\varphi^{h}\right\|_{H^{1}(\Omega)} .
\end{aligned}
$$

Then taking $\varphi^{h}=\mathcal{N}_{\widetilde{q}^{h}}^{h} j-\Pi_{\diamond}^{h} \mathcal{N}_{q} j \in \mathcal{V}_{1, \diamond}^{h}$ and using 2.2., we infer from 4.30 that

$$
\begin{aligned}
\left\|\mathcal{N}_{\widehat{q}^{h}}^{h} j-\Pi_{\diamond}^{h} \mathcal{N}_{q} j\right\|_{H^{1}(\Omega)} & \leq C\left(\left\|q-\widehat{q}^{h}\right\|_{L^{2 s /(s-2)}(\Omega)}+\left\|\mathcal{N}_{q} j-\Pi_{\diamond}^{h} \mathcal{N}_{q} j\right\|_{H^{1}(\Omega)}\right) \\
& \leq C(h|\log h|)^{(s-2) /(2 s)}+C h \leq C(h|\log h|)^{(s-2) /(2 s)},
\end{aligned}
$$

by (4.28)- 4.29). Thus, applying the triangle inequality and 4.28 again, we infer that

$$
\left\|\mathcal{N}_{q} j-\mathcal{N}_{\widetilde{q}^{h}}^{h} j\right\|_{H^{1}(\Omega)} \leq\left\|\mathcal{N}_{q} j-\Pi_{\diamond}^{h} \mathcal{N}_{q} j\right\|_{H^{1}(\Omega)}+\left\|\Pi_{\diamond}^{h} \mathcal{N}_{q} j-\mathcal{N}_{\widetilde{q}^{h}}^{h} j\right\|_{H^{1}(\Omega)} \leq C(h|\log h|)^{(s-2) /(2 s)} .
$$

Similarly, we also get $\left\|\mathcal{D}_{q} g-\mathcal{D}_{\widehat{q}^{h}}^{h} g\right\|_{H^{1}(\Omega)} \leq C(h|\log h|)^{(s-2) /(2 s)}$ and so that

$$
\widehat{\varrho}_{q}^{h}(j, g) \leq C(h|\log h|)^{(s-2) /(2 s)}
$$

for $s$ as in 4.31, which yields the assertion.

With an appropriate a priori choice of the regularization parameter we get convergence under conditions similar to those stated, e.g., in [35] in the Hilbert space setting.

Theorem 4.9. Let $\left(h_{n}\right)_{n},\left(\delta_{n}\right)_{n}$ and $\left(\rho_{n}\right)_{n}$ be any positive sequences such that

$$
\rho_{n} \rightarrow 0, \frac{\delta_{n}}{\sqrt{\rho_{n}}} \rightarrow 0 \text { and } \frac{\widehat{\varrho}_{q}^{h_{n}}\left(j^{\dagger}, g^{\dagger}\right)}{\sqrt{\rho_{n}}} \rightarrow 0 \text { as } n \rightarrow \infty,
$$

where $q$ is any solution to $\mathcal{N}_{q} j^{\dagger}=\mathcal{D}_{q} g^{\dagger}$. Moreover, assume that $\left(j_{\delta_{n}}, g_{\delta_{n}}\right)$ is a sequence satisfying

$$
\left\|j_{\delta_{n}}-j^{\dagger}\right\|_{H^{-1 / 2}(\partial \Omega)}+\left\|g_{\delta_{n}}-g^{\dagger}\right\|_{H^{1 / 2}(\partial \Omega)} \leq \delta_{n}
$$


and that $q_{n}:=q_{\rho_{n}, \delta_{n}}^{h_{n}}$ is an arbitrary minimizer of $\left(\mathcal{P}_{\rho_{n}, \delta_{n}}^{h_{n}}\right)$ for each $n \in \mathbb{N}$. Then a subsequence of $\left(q_{n}\right)$ which is not relabelled and a solution $q^{\dagger}$ to $(\mathcal{I} \mathcal{P})$ exist such that

$$
\lim _{n \rightarrow \infty}\left\|q_{n}-q^{\dagger}\right\|_{L^{1}(\Omega)}=0 \text { and } \lim _{n \rightarrow \infty} \int_{\Omega}\left|\nabla q_{n}\right|=\int_{\Omega}\left|\nabla q^{\dagger}\right| .
$$

Furthermore, $\left(\mathcal{N}_{q_{n}}^{h_{n}} j_{\delta_{n}}\right)$ and $\left(\mathcal{D}_{q_{n}}^{h_{n}} g_{\delta_{n}}\right)$ converge to the unique weak solution $\Phi^{\dagger}=\Phi^{\dagger}\left(q^{\dagger}, j^{\dagger}, g^{\dagger}\right)$ of the boundary value problem (1.1) 1.3 in the $H^{1}(\Omega)$-norm. If $q^{\dagger}$ is unique, then convergence 4.33 holds for the whole sequence.

Uniform $L^{\infty}$ boundedness of $\left(q_{n}\right)$ together with interpolation implies that convergence actually takes place in any $L^{p}$ space with $p \in[1, \infty]$.

Remark 4.10. In case $\mathcal{N}_{q} j^{\dagger}, \mathcal{D}_{q} g^{\dagger} \in H^{2}(\Omega)$ Lemma 4.8 shows that $\widehat{\varrho}_{q}^{h}\left(j^{\dagger}, g^{\dagger}\right) \leq C(h|\log h|)^{r}$ with $r$ as in 4.27). Therefore, in view of 4.32, convergence is obtained if the sequence $\left(\rho_{n}\right)$ is chosen such that

$$
\rho_{n} \rightarrow 0, \frac{\delta_{n}}{\sqrt{\rho_{n}}} \rightarrow 0 \text { and } \frac{\left(h_{n}\left|\log h_{n}\right|\right)^{r}}{\sqrt{\rho_{n}}} \rightarrow 0 \text { as } n \rightarrow \infty .
$$

By regularity theory for elliptic boundary value problems (see, for example, [26, 42]), if $j^{\dagger} \in H^{1 / 2}(\Omega)$, $g^{\dagger} \in H^{3 / 2}(\Omega), q \in C^{0,1}(\Omega), f \in L^{2}(\Omega)$ and either $\partial \Omega$ is $C^{1,1}$-smooth or the domain $\Omega$ is convex, then $\mathcal{N}_{q} j^{\dagger}, \mathcal{D}_{q} g^{\dagger} \in H^{2}(\Omega)$.

Proof of Theorem 4.9. We have from the optimality of $q_{n}$ that

$$
\mathcal{J}_{\delta_{n}}^{h_{n}}\left(q_{n}\right)+\rho_{n} \int_{\Omega} \sqrt{\left|\nabla q_{n}\right|^{2}+\epsilon^{h_{n}}} \leq \mathcal{J}_{\delta_{n}}^{h_{n}}\left(\widehat{q}^{h_{n}}\right)+\rho_{n} \int_{\Omega} \sqrt{\left|\nabla \widehat{q}^{h_{n}}\right|^{2}+\epsilon^{h_{n}}}
$$

where $\widehat{q}^{h_{n}}$ is generated from $q$ according to Lemma 4.6 , and

$$
\begin{aligned}
\mathcal{J}_{\delta_{n}}^{h_{n}}\left(\widehat{q}^{h_{n}}\right)=\int_{\Omega} \widehat{q}^{h_{n}} \nabla\left(\mathcal{N}_{\widehat{q}^{h_{n}}}^{h_{n}} j_{\delta_{n}}-\mathcal{D}_{\widehat{q}^{h_{n}}}^{h_{n}} g_{\delta_{n}}\right) \cdot \nabla\left(\mathcal{N}_{\widehat{q}^{h_{n}}}^{h_{n}} j_{\delta_{n}}-\mathcal{D}_{\widehat{q}^{n_{n}}}^{h_{n}} g_{\delta_{n}}\right) \\
\leq \bar{q}\left\|\mathcal{N}_{\widehat{q}^{h_{n}}}^{h_{n}} j_{\delta_{n}}-\mathcal{D}_{\widehat{q}^{h_{n}}}^{h_{n}} g_{\delta_{n}}\right\|_{H^{1}(\Omega)}^{2} \\
=\bar{q}\left\|\mathcal{N}_{\widehat{q}^{h_{n}}}^{h_{n}} j_{\delta_{n}}-\mathcal{N}_{\widehat{q}^{h_{n}}}^{h_{n}} j^{\dagger}+\mathcal{N}_{\widehat{q}_{n}^{h_{n}}}^{h_{n}} j^{\dagger}-\mathcal{D}_{\widehat{q}^{h_{n}}}^{h_{n}} g^{\dagger}-\mathcal{N}_{q} j^{\dagger}+\mathcal{D}_{q} g^{\dagger}+\mathcal{D}_{\widehat{q}^{h_{n}}}^{h_{n}} g^{\dagger}-\mathcal{D}_{\widehat{q}_{n} h_{n}}^{h_{n}} g_{\delta_{n}}\right\|_{H^{1}(\Omega)}^{2} \\
\leq 4 \bar{q}\left(\left\|\mathcal{N}_{\widehat{q}_{n}^{h_{n}}}^{h_{n}} j_{\delta_{n}}-\mathcal{N}_{\widehat{q}^{h_{n}}}^{h_{n}} j^{\dagger}\right\|_{H^{1}(\Omega)}^{2}+\left\|\mathcal{D}_{\widehat{q}^{h_{n}}}^{h_{n}} g^{\dagger}-\mathcal{D}_{\widehat{q}_{n}}^{h_{n}} g_{\delta_{n}}\right\|_{H^{1}(\Omega)}^{2}\right. \\
\left.\quad+\left\|\mathcal{N}_{\widehat{q}^{h_{n}}}^{h_{n}} j^{\dagger}-\mathcal{N}_{q} j^{\dagger}\right\|_{H^{1}(\Omega)}^{2}+\left\|\mathcal{D}_{\widehat{q}^{h_{n}}}^{h_{n}} g^{\dagger}-\mathcal{D}_{q} g^{\dagger}\right\|_{H^{1}(\Omega)}^{2}\right) \\
\leq C\left(\left\|j_{\delta_{n}}-j^{\dagger}\right\|_{H^{-1 / 2}(\partial \Omega)}^{2}+\left\|g_{\delta_{n}}-g^{\dagger}\right\|_{H^{-1 / 2}(\partial \Omega)}^{2}\right)+C \widehat{\varrho}_{q}^{h_{n}}\left(j^{\dagger}, g^{\dagger}\right)^{2} \\
\leq C\left(\delta_{n}^{2}+\widehat{\varrho}_{q}^{h_{n}}\left(j^{\dagger}, g^{\dagger}\right)^{2}\right),
\end{aligned}
$$

where we have used Lemma 4.2 and the fact $\mathcal{N}_{q} j^{\dagger}=\mathcal{D}_{q} g^{\dagger}$. Moreover, by Lemma 4.6, we have that

$$
\lim _{n \rightarrow \infty} \int_{\Omega} \sqrt{\left|\nabla \widehat{q}^{h_{n}}\right|^{2}+\epsilon^{h_{n}}}=\lim _{n \rightarrow \infty} \int_{\Omega}\left|\nabla \widehat{q}^{h_{n}}\right|=\int_{\Omega}|\nabla q| .
$$

We therefore conclude from 4.34 and 4.32 that

$$
\lim _{n \rightarrow \infty} \frac{\mathcal{J}_{\delta_{n}}^{h_{n}}\left(\widehat{q}^{h_{n}}\right)}{\rho_{n}}=0, \quad \lim _{n \rightarrow \infty} \mathcal{J}_{\delta_{n}}^{h_{n}}\left(q_{n}\right)=0
$$

and

$$
\limsup _{n \rightarrow \infty} \int_{\Omega}\left|\nabla q_{n}\right| \leq \limsup _{n \rightarrow \infty} \int_{\Omega} \sqrt{\left|\nabla q_{n}\right|^{2}+\epsilon^{h_{n}}} \leq \limsup _{n \rightarrow \infty} \int_{\Omega} \sqrt{\left|\nabla \widehat{q}^{h_{n}}\right|^{2}+\epsilon^{h_{n}}}=\int_{\Omega}|\nabla q| .
$$


Thus, $\left(q_{n}\right)$ is bounded in the $B V(\Omega)$-norm. A subsequence which is denoted by the same symbol and an element $q^{\dagger} \in \mathcal{Q}_{a d}$ exist such that $\left(q_{n}\right)$ converges to $q^{\dagger}$ in the $L^{1}(\Omega)$-norm and

$$
\int_{\Omega}\left|\nabla q^{\dagger}\right| \leq \liminf _{n \rightarrow \infty} \int_{\Omega}\left|\nabla q_{n}\right|
$$

Using Lemma 4.2 again, we infer that

$$
\begin{aligned}
\| \mathcal{N}_{q_{n}}^{h_{n}} j^{\dagger} & -\mathcal{D}_{q_{n}}^{h_{n}} g^{\dagger} \|_{H^{1}(\Omega)}^{2} \\
& \leq 3\left(\left\|\mathcal{N}_{q_{n}}^{h_{n}} j^{\dagger}-\mathcal{N}_{q_{n}}^{h_{n}} j_{\delta_{n}}\right\|_{H^{1}(\Omega)}^{2}+\left\|\mathcal{D}_{q_{n}}^{h_{n}} g^{\dagger}-\mathcal{D}_{q_{n}}^{h_{n}} g_{\delta_{n}}\right\|_{H^{1}(\Omega)}^{2}+\left\|\mathcal{N}_{q_{n}}^{h_{n}} j_{\delta_{n}}-\mathcal{D}_{q_{n}}^{h_{n}} g_{\delta_{n}}\right\|_{H^{1}(\Omega)}^{2}\right) \\
& \leq C \delta_{n}^{2}+3\left\|\mathcal{N}_{q_{n}}^{h_{n}} j_{\delta_{n}}-\mathcal{D}_{q_{n}}^{h_{n}} g_{\delta_{n}}\right\|_{H^{1}(\Omega)}^{2} \\
& \leq C\left(\delta_{n}^{2}+\mathcal{J}_{\delta_{n}}^{h_{n}}\left(q_{n}\right)\right) .
\end{aligned}
$$

Thus, using Lemma 4.3 we obtain from the last inequality and 4.36 that

$$
\left\|\mathcal{N}_{q^{\dagger}} j^{\dagger}-\mathcal{D}_{q^{\dagger}} g^{\dagger}\right\|_{H^{1}(\Omega)}^{2}=\lim _{n \rightarrow \infty}\left\|\mathcal{N}_{q_{n}}^{h_{n}} j^{\dagger}-\mathcal{D}_{q_{n}}^{h_{n}} g^{\dagger}\right\|_{H^{1}(\Omega)}^{2}=0
$$

and so that

$$
\mathcal{N}_{q^{\dagger}} j^{\dagger}=\mathcal{D}_{q^{\dagger}} g^{\dagger}, \text { i.e., } q^{\dagger} \in \mathcal{I}_{\mathcal{Q}_{a d}}\left(j^{\dagger}, g^{\dagger}\right) .
$$

Furthermore, it follows from 4.37)-4.38 that

$$
\int_{\Omega}\left|\nabla q^{\dagger}\right| \leq \liminf _{n \rightarrow \infty} \int_{\Omega}\left|\nabla q_{n}\right| \leq \limsup _{n \rightarrow \infty} \int_{\Omega}\left|\nabla q_{n}\right| \leq \int_{\Omega}|\nabla q|,
$$

for any solution $q$ to $\mathcal{N}_{q} j^{\dagger}=\mathcal{D}_{q} g^{\dagger}$, hence, in view of 4.39, $q^{\dagger}$ is a total variation minimizing solution of the identification problem, i.e., a solution to $(\mathcal{I P})$. Moreover, by setting $q=q^{\dagger}$, we get

$$
\int_{\Omega}\left|\nabla q^{\dagger}\right|=\lim _{n \rightarrow \infty} \int_{\Omega}\left|\nabla q_{n}\right|
$$

Finally, Lemma 4.3 shows that the sequence $\left(\mathcal{N}_{q_{n}}^{h_{n}} j_{\delta_{n}}, \mathcal{D}_{q_{n}}^{h_{n}} g_{\delta_{n}}\right)$ converges in the $H^{1}(\Omega) \times H^{1}(\Omega)$-norm to $\left(\mathcal{N}_{q^{\dagger}} j^{\dagger}, \mathcal{D}_{q^{\dagger}} g^{\dagger}\right)$, where $\Phi^{\dagger}:=\mathcal{N}_{q^{\dagger}} j^{\dagger}=\mathcal{D}_{q^{\dagger}} g^{\dagger}$ is the unique weak solution of the elliptic system 1.1 - 1.3), which finishes the proof.

\section{Projected Armijo algorithm and numerical test}

In this section we present the projected Armijo algorithm (see [27, Chapter 5]) for numerically solving the minimization problem $\left(\mathcal{P}_{\rho, \delta}^{h}\right)$. We note that many other efficient solution methods are available, see for example [8].

\subsection{Projected Armijo algorithm}

\subsubsection{Differentiability of the cost functional}

Similarly to Lemma 2.3 one also sees that the discrete Neumann and Dirichlet operators $\mathcal{N}^{h}, \mathcal{D}^{h}$ are Fréchet differentiable on the set $\mathcal{Q}$. For given $j_{\delta} \in H^{-1 / 2}(\partial \Omega)$ and each $q \in \mathcal{Q}$ the Fréchet derivative $\mathcal{N}^{h^{\prime}}(q) \xi=$ : $\mathcal{N}_{q}^{h^{\prime}} j_{\delta}(\xi)$ in the direction $\xi \in L^{\infty}(\Omega)$ is an element of $\mathcal{V}_{1, \diamond}^{h}$ and satisfies the equation

$$
\int_{\Omega} q \nabla \mathcal{N}_{q}^{h^{\prime}} j_{\delta}(\xi) \cdot \nabla \varphi^{h}=-\int_{\Omega} \xi \nabla \mathcal{N}_{q}^{h} j_{\delta} \cdot \nabla \varphi^{h}
$$


for all $\varphi^{h} \in \mathcal{V}_{1, \diamond}^{h}$. Likewise, for fixed $g_{\delta} \in H^{1 / 2}(\partial \Omega)$ and each $q \in \mathcal{Q}$ the Fréchet derivative $\mathcal{D}^{h^{\prime}}(q) \xi=$ : $\mathcal{D}_{q}^{h^{\prime}} g_{\delta}(\xi)$ in the direction $\xi \in L^{\infty}(\Omega)$ is an element of $\mathcal{V}_{1,0}^{h}$ and satisfies the equation

$$
\int_{\Omega} q \nabla \mathcal{D}_{q}^{h^{\prime}} g_{\delta}(\xi) \cdot \nabla \psi^{h}=-\int_{\Omega} \xi \nabla \mathcal{D}_{q}^{h} g_{\delta} \cdot \nabla \psi^{h}
$$

for all $\psi^{h} \in \mathcal{V}_{1,0}^{h}$.

The functional $\mathcal{J}_{\delta}^{h}$ is therefore Fréchet differentiable on the set $\mathcal{Q}$. For each $q \in \mathcal{Q}$ the action of the Fréchet derivative in the direction $\xi \in L^{\infty}(\Omega)$ is given by

$$
\begin{aligned}
\mathcal{J}_{\delta}^{h^{\prime}}(q)(\xi)= & \int_{\Omega} \xi \nabla\left(\mathcal{N}_{q}^{h} j_{\delta}-\mathcal{D}_{q}^{h} g_{\delta}\right) \cdot \nabla\left(\mathcal{N}_{q}^{h} j_{\delta}-\mathcal{D}_{q}^{h} g_{\delta}\right) \\
& +2 \int_{\Omega} q \nabla\left(\mathcal{N}_{q}^{h^{\prime}} j_{\delta}(\xi)-\mathcal{D}_{q}^{h^{\prime}} g_{\delta}(\xi)\right) \cdot \nabla\left(\mathcal{N}_{q}^{h} j_{\delta}-\mathcal{D}_{q}^{h} g_{\delta}\right) \\
= & \int_{\Omega} \xi \nabla\left(\mathcal{N}_{q}^{h} j_{\delta}-\mathcal{D}_{q}^{h} g_{\delta}\right) \cdot \nabla\left(\mathcal{N}_{q}^{h} j_{\delta}-\mathcal{D}_{q}^{h} g_{\delta}\right) \\
& +2 \int_{\Omega} q \nabla \mathcal{N}_{q}^{h^{\prime}} j_{\delta}(\xi) \cdot \nabla\left(\mathcal{N}_{q}^{h} j_{\delta}-\mathcal{D}_{q}^{h} g_{\delta}\right)-2 \int_{\Omega} q \nabla \mathcal{N}_{q}^{h} j_{\delta} \cdot \nabla \mathcal{D}_{q}^{h^{\prime}} g_{\delta}(\xi) \\
& +2 \int_{\Omega} q \nabla \mathcal{D}_{q}^{h} g_{\delta} \cdot \nabla \mathcal{D}_{q}^{h^{\prime}} g_{\delta}(\xi) .
\end{aligned}
$$

Since $\mathcal{N}_{q}^{h} j_{\delta}, \mathcal{D}_{q}^{h} g_{\delta} \in \mathcal{V}_{1, \diamond}^{h}$ and $\mathcal{D}_{q}^{h^{\prime}} g_{\delta}(\xi) \in \mathcal{V}_{1,0}^{h} \subset \mathcal{V}_{1, \diamond}^{h}$, it follows from 5.1, 3.6 and 3.8 that

$$
\begin{aligned}
& \int_{\Omega} q \nabla \mathcal{N}_{q}^{h^{\prime}} j_{\delta}(\xi) \cdot \nabla\left(\mathcal{N}_{q}^{h} j_{\delta}-\mathcal{D}_{q}^{h} g_{\delta}\right)-\int_{\Omega} q \nabla \mathcal{N}_{q}^{h} j_{\delta} \cdot \nabla \mathcal{D}_{q}^{h^{\prime}} g_{\delta}(\xi)+\int_{\Omega} q \nabla \mathcal{D}_{q}^{h} g_{\delta} \cdot \nabla \mathcal{D}_{q}^{h^{\prime}} g_{\delta}(\xi) \\
& \quad=-\int_{\Omega} \xi \nabla \mathcal{N}_{q}^{h} j_{\delta} \cdot \nabla\left(\mathcal{N}_{q}^{h} j_{\delta}-\mathcal{D}_{q}^{h} g_{\delta}\right)-\left\langle j_{\delta}, \gamma \mathcal{D}_{q}^{h^{\prime}} g_{\delta}(\xi)\right\rangle-\left(f, \mathcal{D}_{q}^{h^{\prime}} g_{\delta}(\xi)\right)+\left(f, \mathcal{D}_{q}^{h^{\prime}} g_{\delta}(\xi)\right) \\
& \quad=-\int_{\Omega} \xi \nabla \mathcal{N}_{q}^{h} j_{\delta} \cdot \nabla\left(\mathcal{N}_{q}^{h} j_{\delta}-\mathcal{D}_{q}^{h} g_{\delta}\right)
\end{aligned}
$$

and so that

$$
\begin{aligned}
\mathcal{J}_{\delta}^{h^{\prime}}(q)(\xi) & =\int_{\Omega} \xi \nabla\left(\mathcal{N}_{q}^{h} j_{\delta}-\mathcal{D}_{q}^{h} g_{\delta}\right) \cdot \nabla\left(\mathcal{N}_{q}^{h} j_{\delta}-\mathcal{D}_{q}^{h} g_{\delta}\right)-2 \int_{\Omega} \xi \nabla \mathcal{N}_{q}^{h} j_{\delta} \cdot \nabla\left(\mathcal{N}_{q}^{h} j_{\delta}-\mathcal{D}_{q}^{h} g_{\delta}\right) \\
& =\int_{\Omega} \xi\left(\nabla \mathcal{D}_{q}^{h} g_{\delta} \cdot \nabla \mathcal{D}_{q}^{h} g_{\delta}-\nabla \mathcal{N}_{q}^{h} j_{\delta} \cdot \nabla \mathcal{N}_{q}^{h} j_{\delta}\right) .
\end{aligned}
$$

Therefore, the derivative of the cost functional $\Upsilon_{\rho, \delta}^{h}$ of $\left(\mathcal{P}_{\rho, \delta}^{h}\right)$ at $q \in \mathcal{Q}_{a d}^{h}$ in the direction $\xi \in \mathcal{V}_{1}^{h}$ is given by

$$
\Upsilon_{\rho, \delta}^{h}{ }^{\prime}(q)(\xi)=\int_{\Omega} \xi\left(\nabla \mathcal{D}_{q}^{h} g_{\delta} \cdot \nabla \mathcal{D}_{q}^{h} g_{\delta}-\nabla \mathcal{N}_{q}^{h} j_{\delta} \cdot \nabla \mathcal{N}_{q}^{h} j_{\delta}\right)+\rho \int_{\Omega} \frac{\nabla q \cdot \nabla \xi}{\sqrt{|\nabla q|^{2}+\epsilon^{h}}}
$$

Let $\left\{N_{j}: j=1, \ldots, M^{h}\right\}$ be the set of nodes of the triangulation $\mathcal{T}^{h}$, then $\mathcal{V}_{1}^{h}$ is a finite dimensional vector space with dimension $M^{h}$. Let $\left\{\phi_{1}, \ldots, \phi_{M^{h}}\right\}$ be the basis of $\mathcal{V}_{1}^{h}$ consisting hat functions, i.e. $\phi_{i}\left(N_{j}\right)=\delta_{i j}$ for all $1 \leq i, j \leq M^{h}$, where $\delta_{i j}$ is the Kronecker symbol. Each functional $u \in \mathcal{V}_{1}^{h}$ then can be identified with a vector $\left(u_{1}, \ldots, u_{M^{h}}\right) \in \mathbb{R}^{M^{h}}$ consisting of the nodal values of $u$, i.e.

$$
u=\sum_{j=1}^{M^{h}} u_{j} \phi_{j} \text { with } u_{j}=u\left(N_{j}\right)
$$

In $\mathcal{V}_{1}^{h}$ we use the Euclidean inner product $\langle\cdot, \cdot\rangle_{E}$. For each $u=\left(u_{1}, \ldots, u_{M^{h}}\right)$ and $v=\left(v_{1}, \ldots, v_{M^{h}}\right)$, we have $\langle u, v\rangle_{E}=\sum_{j=1}^{M^{h}} u_{j} v_{j}$. Let us denote the gradient of $\Upsilon_{\rho, \delta}^{h}$ at $q \in \mathcal{Q}_{a d}^{h}$ by $\nabla \Upsilon_{\rho, \delta}^{h}(q)=\left(\Upsilon_{1}, \ldots, \Upsilon_{M^{h}}\right)$. We then have from $(5.3)$ with $\xi=\left(\xi_{1}, \ldots, \xi_{M^{h}}\right) \in \mathcal{V}_{1}^{h}$ that

$$
\sum_{j=1}^{M^{h}} \xi_{j} \int_{\Omega}\left(\phi_{j}\left(\nabla \mathcal{D}_{q}^{h} g_{\delta} \cdot \nabla \mathcal{D}_{q}^{h} g_{\delta}-\nabla \mathcal{N}_{q}^{h} j_{\delta} \cdot \nabla \mathcal{N}_{q}^{h} j_{\delta}\right)+\frac{\rho \nabla q \cdot \nabla \phi_{j}}{\sqrt{|\nabla q|^{2}+\epsilon^{h}}}\right)=\sum_{j=1}^{M^{h}} \xi_{j} \Upsilon_{j}
$$


which yields

$$
\Upsilon_{j}=\int_{\Omega} \phi_{j}\left(\nabla \mathcal{D}_{q}^{h} g_{\delta} \cdot \nabla \mathcal{D}_{q}^{h} g_{\delta}-\nabla \mathcal{N}_{q}^{h} j_{\delta} \cdot \nabla \mathcal{N}_{q}^{h} j_{\delta}\right)+\rho \int_{\Omega} \frac{\nabla q \cdot \nabla \phi_{j}}{\sqrt{|\nabla q|^{2}+\epsilon^{h}}}
$$

for all $j=1, \ldots, M^{h}$.

\subsubsection{Algorithm}

The projected Armijo algorithm is then read as: given a step size control $\beta \in(0,1)$, an initial approximation $q_{0}^{h} \in \mathcal{Q}_{a d}^{h}$, a smoothing parameter $\epsilon^{h}$, number of iteration $N$ and setting $k=0$.

1. Compute $\mathcal{N}_{q_{k}^{h}}^{h} j_{\delta}$ and $\mathcal{D}_{q_{k}^{h}}^{h} g_{\delta}$ from the variational equations

$$
\int_{\Omega} q_{k}^{h} \nabla \mathcal{N}_{q_{k}^{h}}^{h} j_{\delta} \cdot \nabla \varphi^{h}=\left\langle j_{\delta}, \gamma \varphi^{h}\right\rangle+\left(f, \varphi^{h}\right) \text { for all } \varphi^{h} \in \mathcal{V}_{1, \diamond}^{h}
$$

and

$$
\int_{\Omega} q_{k}^{h} \nabla \mathcal{D}_{q_{k}^{h}}^{h} g_{\delta} \cdot \nabla \psi^{h}=\left(f, \psi^{h}\right) \text { for all } \psi^{h} \in \mathcal{V}_{1,0}^{h},
$$

respectively, as well as $\Upsilon_{\rho, \delta}^{h}\left(q_{k}^{h}\right)$ according to 3.12, 3.13.

2. Compute the gradient $\nabla \Upsilon_{\rho, \delta}^{h}\left(q_{k}^{h}\right)$ with the $j^{\text {th }}$-component given by

$$
\Upsilon_{j}=\int_{\Omega} \phi_{j}\left(\nabla \mathcal{D}_{q_{k}^{h}}^{h} g_{\delta} \cdot \nabla \mathcal{D}_{q_{k}^{h}}^{h} g_{\delta}-\nabla \mathcal{N}_{q_{k}^{h}}^{h} j_{\delta} \cdot \nabla \mathcal{N}_{q_{k}^{h}}^{h} j_{\delta}\right)+\rho \int_{\Omega} \frac{\nabla q_{k}^{h} \cdot \nabla \phi_{j}}{\sqrt{\left|\nabla q_{k}^{h}\right|^{2}+\epsilon^{h}}},
$$

due to 5.4.

3. Set $G_{k}^{h}:=\sum_{j=1}^{M^{h}} \Upsilon_{j} \phi_{j}$.

(a) Compute

$$
\tilde{q}_{k}^{h}:=\max \left(\underline{q}, \min \left(q_{k}^{h}-\beta G_{k}^{h}, \bar{q}\right)\right),
$$

$\mathcal{N}_{\tilde{q}_{k}^{h}}^{h} j_{\delta}, \mathcal{D}_{\tilde{q}_{k}^{h}}^{h} g_{\delta}$, according to 5.5, 5.6), $\Upsilon_{\rho, \delta}^{h}\left(\tilde{q}_{k}^{h}\right)$, according to 3.12, 3.13, and $L:=\Upsilon_{\rho, \delta}^{h}\left(\tilde{q}_{k}^{h}\right)-\Upsilon_{\rho, \delta}^{h}\left(q_{k}^{h}\right)+\tau \beta\left\|\tilde{q}_{k}^{h}-q_{k}^{h}\right\|_{L^{2}(\Omega)}^{2}$ with $\tau=10^{-4}$.

(b) If $L \leq 0$

else

go to the next step (c) below

set $\beta:=\frac{\beta}{2}$ and then go back (a)

(c) Update $q_{k}^{h}=\tilde{q}_{k}^{h}$, set $k=k+1$.

4. Compute

$$
\text { Tolerance }:=\left\|\nabla \Upsilon_{\rho, \delta}^{h}\left(q_{k}^{h}\right)\right\|_{L^{2}(\Omega)}-\tau_{1}-\tau_{2}\left\|\nabla \Upsilon_{\rho, \delta}^{h}\left(q_{0}^{h}\right)\right\|_{L^{2}(\Omega)}
$$

with $\tau_{1}:=10^{-3} h^{1 / 2}$ and $\tau_{2}:=10^{-2} h^{1 / 2}$. If Tolerance $\leq 0$ or $k>N$, then stop; otherwise go back Step 1 . 


\section{$5.2 \quad$ Numerical tests}

We now illustrate the theoretical result with numerical examples. For this purpose we consider the the boundary value problem

$$
\begin{aligned}
-\nabla \cdot\left(q^{\dagger} \nabla \Phi\right) & =f \text { in } \Omega, \\
q^{\dagger} \nabla \Phi \cdot \vec{n} & =j^{\dagger} \text { on } \partial \Omega \text { and } \\
\Phi & =g^{\dagger} \text { on } \partial \Omega
\end{aligned}
$$

with $\Omega=\left\{x=\left(x_{1}, x_{2}\right) \in \mathbb{R}^{2} \mid-1<x_{1}, x_{2}<1\right\}$. The special constants $\underline{q}$ and $\bar{q}$ in the definition of the set $\mathcal{Q}$ according to 1.4 are respectively chosen as 0.05 and 10 .

We assume that the known source $f$ is discontinuous and given by

$$
f=\frac{3}{2} \chi_{D}-\frac{1}{2} \chi_{\Omega \backslash D}
$$

where $\chi_{D}$ is the characteristic function of $D:=\left\{\left(x_{1}, x_{2}\right) \in \Omega|| x_{1} \mid \leq 1 / 2\right.$ and $\left.\left|x_{2}\right| \leq 1 / 2\right\}$. Note that $(f, 1)=0$, so that $c_{f}=0$. The sought conductivity $q^{\dagger}$ in the equation (5.8)-(5.9) is assumed to be discontinuous and given by

$$
q^{\dagger}=3 \chi_{\Omega_{1}}+2 \chi_{\Omega_{2}}+\chi_{\Omega \backslash\left(\Omega_{1} \cup \Omega_{2}\right)},
$$

where

$$
\begin{aligned}
& \Omega_{1}:=\left\{\left(x_{1}, x_{2}\right) \in \Omega \mid 9\left(x_{1}+1 / 2\right)^{2}+16\left(x_{2}-1 / 2\right)^{2} \leq 1\right\} \text { and } \\
& \Omega_{2}:=\left\{\left(x_{1}, x_{2}\right) \in \Omega \mid\left(x_{1}-1 / 2\right)^{2}+\left(x_{2}+1 / 2\right)^{2} \leq 1 / 16\right\} .
\end{aligned}
$$

For the discretization we divide the interval $(-1,1)$ into $\ell$ equal segments and so that the domain $\Omega=(-1,1)^{2}$ is divided into $2 \ell^{2}$ triangles, where the diameter of each triangle is $h_{\ell}=\frac{\sqrt{8}}{\ell}$. In the minimization problem $\left(\begin{array}{c}\mathcal{P}_{\rho, \delta}^{h} \\ )\end{array}\right)$ we take $h=h_{\ell}$ and $\rho=\rho_{\ell}=0.01 \sqrt{h_{\ell}}$. We use the projected Armijo algorithm which is described in Subsection 5.1 for computing the numerical solution of the problem $\left(\mathcal{P}_{\rho_{\ell}, \delta_{\ell}}^{h_{\ell}}\right)$. The step size control is chosen with $\beta=0.75$ while the smoothing parameter $\epsilon^{h_{\ell}}=\rho_{\ell}$. The initial approximation is the constant function defined by $q_{0}^{h_{\ell}}=1.5$.

Example 5.1. In this example the Neumann boundary condition $j^{\dagger} \in H_{-c_{f}}^{-1 / 2}(\partial \Omega)$ in the equation (5.9) is chosen to be the piecewise constant function defined by

$$
\begin{aligned}
j^{\dagger}= & \chi_{(0,1] \times\{-1\}}-\chi_{[-1,0] \times\{1\}}+2 \chi_{(0,1] \times\{1\}}-2 \chi_{[-1,0] \times\{-1\}} \\
& +3 \chi_{\{-1\} \times(-1,0]}-3 \chi_{\{1\} \times(0,1)}+4 \chi_{\{1\} \times(-1,0]}-4 \chi_{\{-1\} \times(0,1)} .
\end{aligned}
$$

so that $\left\langle j^{\dagger}, 1\right\rangle=0$. The Dirichlet boundary condition $g^{\dagger} \in H_{\diamond}^{1 / 2}(\partial \Omega)$ in the equation $(5.10)$ is then defined as $g^{\dagger}=\gamma \mathcal{N}_{q^{\dagger}} j^{\dagger}$, where $\mathcal{N}_{q^{\dagger}} j^{\dagger}$ is the unique weak solution to the Neumann problem 5.8-5.9). For the numerical solution of the pure Neumann problem (5.8)-(5.9) we use the penalty technique, see e.g. [9, 25] for more details. Furthermore, to avoid a so-called inverse crime, we generate the data on a finer grid than those used in the computations. To do so, we first solve the Neumann problem (5.8)-(5.9) on the very fine grid $\ell=128$, and then handle $\left(j^{\dagger}, g^{\dagger}\right)$ on this grid for our computational process below.

We assume that noisy observations are available in the form

$$
\left(j_{\delta_{\ell}}, g_{\delta_{\ell}}\right)=\left(j^{\dagger}+\theta_{\ell} \cdot R_{j^{\dagger}}, g^{\dagger}+\theta_{\ell} \cdot R_{g^{\dagger}}\right) \text { for some } \theta_{\ell}>0 \text { depending on } \ell,
$$

where $R_{j^{\dagger}}$ and $R_{g^{\dagger}}$ are $\partial M^{h_{\ell}} \times 1$-matrices of random numbers on the interval $(-1,1)$ which are generated by the MATLAB function "rand" and $\partial M^{h_{\ell}}$ is the number of boundary nodes of the triangulation $\mathcal{T}^{h_{\ell}}$. The measurement error is then computed as $\delta_{\ell}=\left\|j_{\delta_{\ell}}-j^{\dagger}\right\|_{L^{2}(\partial \Omega)}+\left\|g_{\delta_{\ell}}-g^{\dagger}\right\|_{L^{2}(\partial \Omega)}$. To satisfy the condition $\delta_{\ell} \cdot \rho_{\ell}^{-1 / 2} \rightarrow 0$ as $\ell \rightarrow \infty$ in Theorem 4.9 we below take $\theta_{\ell}=h_{\ell} \sqrt{\rho_{\ell}}$. In doing so, we reversely mimic the 
situation of a given sequence of noise levels $\delta_{\ell}$ tending to zero and of choosing the discretization level as well as the regularization parameter in dependence of the noise level.

Our computational process will be started with the coarsest level $\ell=4$. In each iteration $k$ we compute Tolerance defined by (5.7). Then the iteration is stopped if Tolerance $\leq 0$ or the number of iterations reaches the maximum iteration count of 1000. After obtaining the numerical solution of the first iteration process with respect to the coarsest level $\ell=4$, we use its interpolation on the next finer mesh $\ell=8$ as an initial approximation $q_{0}^{h_{\ell}}$ for the algorithm on this finer mesh, and so on for $\ell=16,32,64$.

Let $q_{\ell}$ denote the conductivity obtained at the final iterate of the algorithm corresponding to the refinement level $\ell$. Furthermore, let $\mathcal{N}_{q_{\ell}}^{h_{\ell}} j_{\delta_{\ell}}$ and $\mathcal{D}_{q_{\ell}}^{h_{\ell}} g_{\delta_{\ell}}$ denote the computed numerical solution to the Neumann problem

$$
-\nabla \cdot\left(q_{\ell} \nabla u\right)=f \text { in } \Omega \text { and } q_{\ell} \nabla u \cdot \vec{n}=j_{\delta_{\ell}} \text { on } \partial \Omega
$$

and the Dirichlet problem

$$
-\nabla \cdot\left(q_{\ell} \nabla v\right)=f \text { in } \Omega \text { and } v=g_{\ell} \text { on } \partial \Omega,
$$

respectively. The notations $\mathcal{N}_{q^{\dagger}}^{h_{\ell}} j^{\dagger}$ and $\mathcal{D}_{q^{\dagger}}^{h_{\ell}} g^{\dagger}$ of the exact numerical solutions are to be understood similarly. We use the following abbreviations for the errors

$$
L_{q}^{2}=\left\|q_{\ell}-q^{\dagger}\right\|_{L^{2}(\Omega)}, \quad L_{\mathcal{N}}^{2}=\left\|\mathcal{N}_{q_{\ell}}^{h_{\ell}} j_{\delta_{\ell}}-\mathcal{N}_{q^{\dagger}}^{h_{\ell}} j^{\dagger}\right\|_{L^{2}(\Omega)} \quad \text { and } \quad L_{\mathcal{D}}^{2}=\left\|\mathcal{D}_{q_{\ell}}^{h_{\ell}} g_{\delta_{\ell}}-\mathcal{D}_{q^{\dagger}}^{h_{\ell}} g^{\dagger}\right\|_{L^{2}(\Omega)} .
$$

The numerical results are summarized in Table 1 and Table 2, where we present the refinement level $\ell$, the mesh size $h_{\ell}$ of the triangulation, the regularization parameter $\rho_{\ell}$, the measurement noise $\delta_{\ell}$, the number of iterations, the value of Tolerance, the errors $L_{q}^{2}, L_{\mathcal{N}}^{2}, L_{\mathcal{D}}^{2}$, and their experimental order of convergence (EOC) defined by

$$
\mathrm{EOC}_{\Xi}:=\frac{\ln \Xi\left(h_{1}\right)-\ln \Xi\left(h_{2}\right)}{\ln h_{1}-\ln h_{2}}
$$

with $\Xi(h)$ being an error functional with respect to the mesh size $h$. The convergence history given in Table 1 and Table 2 shows that the projected Armijo algorithm performs well for our identification problem.

All figures presented hereafter correspond to the finest level $\ell=64$. Figure 1 from left to right shows the interpolation $I_{1}^{h_{\ell}} q^{\dagger}$, the numerical solution $q_{\ell}$ computed by the algorithm at the $953^{\text {th }}$ iteration, and the differences $\mathcal{N}_{q_{\ell}}^{h_{\ell}} j_{\delta_{\ell}}-\mathcal{N}_{q^{\dagger}}^{h_{\ell}} j^{\dagger}$ and $\mathcal{D}_{q_{\ell}}^{h_{\ell}} g_{\delta_{\ell}}-\mathcal{D}_{q^{\dagger}}^{h_{\ell}} g^{\dagger}$.

\begin{tabular}{|c|c|c|c|c|c|c|}
\hline \multicolumn{7}{|c|}{ Convergence history and EOC } \\
\hline$\ell$ & $L_{q}^{2}$ & $L_{\mathcal{N}}^{2}$ & $L_{\mathcal{D}}^{2}$ & $\mathrm{EOC}_{L_{q}^{2}}$ & $\mathrm{EOC}_{L_{\mathcal{N}}^{2}}$ & $\mathrm{EOC}_{L_{\mathcal{D}}^{2}}$ \\
\hline 4 & 0.7906 & 0.3016 & 0.1371 & -9 & $-N$ & - \\
\hline 8 & 0.4768 & 0.1546 & $6.2771 \mathrm{e}-2$ & 0.7296 & 0.9637 & 1.1271 \\
\hline 16 & 0.2306 & $6.9702 \mathrm{e}-2$ & $2.1228 \mathrm{e}-2$ & 1.0480 & 1.1497 & 1.5641 \\
\hline 32 & 0.1271 & $3.0668 \mathrm{e}-2$ & $9.9234 \mathrm{e}-3$ & 0.8594 & 1.1845 & 1.0971 \\
\hline 64 & $6.7791 \mathrm{e}-2$ & $1.2116 \mathrm{e}-2$ & $5.1055 \mathrm{e}-3$ & 0.9068 & 1.3398 & 0.9588 \\
\hline \multicolumn{4}{|c|}{ Mean of EOC } & 0.8859 & 1.1594 & 1.1868 \\
\hline
\end{tabular}

\begin{tabular}{|c|l|l|l|r|c|}
\hline \multicolumn{7}{|c|}{ Convergence history } \\
\hline$\ell$ & $h_{\ell}$ & $\rho_{\ell}$ & $\delta_{\ell}$ & Iterate & Tolerance \\
\hline 4 & 0.7071 & $8.4091 \mathrm{e}-3$ & 0.1733 & 1000 & 0.2459 \\
\hline 8 & 0.3536 & $5.9460 \mathrm{e}-3$ & $8.4273 \mathrm{e}-2$ & 1000 & $3.2771 \mathrm{e}-2$ \\
\hline 16 & 0.1766 & $4.2045 \mathrm{e}-3$ & $3.4320 \mathrm{e}-2$ & 1000 & $5.8479 \mathrm{e}-3$ \\
\hline 32 & $8.8388 \mathrm{e}-2$ & $2.9730 \mathrm{e}-3$ & $1.5877 \mathrm{e}-2$ & 1000 & $9.4359 \mathrm{e}-5$ \\
\hline 64 & $4.4194 \mathrm{e}-2$ & $2.1022 \mathrm{e}-3$ & $6.7743 \mathrm{e}-3$ & 953 & $-7.6116 \mathrm{e}-5$ \\
\hline
\end{tabular}

Table 1: Refinement level $\ell$, mesh size $h_{\ell}$ of the triangulation, regularization parameter $\rho_{\ell}$, measurement noise $\delta_{\ell}$, number of iterates and value of Tolerance.

Table 2: Errors $L_{q}^{2}, L_{\mathcal{N}}^{2}, L_{\mathcal{D}}^{2}$, and their EOC between finest and coarsest level. 

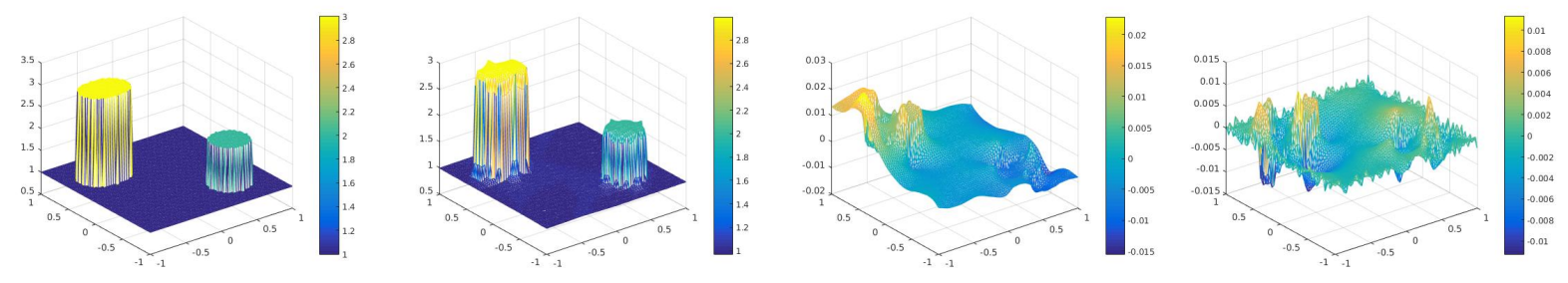

Figure 1: Interpolation $I_{1}^{h_{\ell}} q^{\dagger}$, computed numerical solution $q_{\ell}$ of the algorithm at the $953^{\text {th }}$ iteration, and the differences $\mathcal{N}_{q_{\ell}}^{h_{\ell}} j_{\delta_{\ell}}-\mathcal{N}_{q^{\dagger}}^{h_{\ell}} j^{\dagger}$ and $\mathcal{D}_{q_{\ell}}^{h_{\ell}} g_{\delta_{\ell}}-\mathcal{D}_{q^{\dagger}}^{h_{\ell}} g^{\dagger}$, for $\ell=64, \delta_{\ell}=6.7743 e-3$.

We observe a decrease of all errors as the noise level gets smaller, as expected from our convergence result, however, with respect to different norms. In particular, in our computations we use an $L^{2}$ noise level, as realistic in applications.

Example 5.2. In this example we consider noisy observations in the form

$$
\left(j_{\delta_{\ell}}, g_{\delta_{\ell}}\right)=\left(j^{\dagger}+\theta \cdot R_{j^{\dagger}}, g^{\dagger}+\theta \cdot R_{g^{\dagger}}\right),
$$

where $j^{\dagger}$ is defined by (5.11). This is different from 5.12 , since here $\theta>0$ is independent of $\ell$.

Using the computational process which was described as in Example 5.1 starting with $\ell=4$, in Table 3 we perform the numerical results for the finest grid $\ell=64$ and with different values of $\theta$.

\begin{tabular}{|l|l|l|l|l|l|l|}
\hline \multicolumn{7}{|c|}{ Numerical results for the finest grid $\ell=64$} \\
\hline$\theta$ & $\delta_{\ell}$ & Iterate & Tolerance & $L_{q}^{2}$ & $L_{\mathcal{N}}^{2}$ & $L_{\mathcal{D}}^{2}$ \\
\hline 0.005 & 0.0167 & 991 & $-9.7239 \mathrm{e}-5$ & $7.7012 \mathrm{e}-2$ & $1.3085 \mathrm{e}-2$ & $7.5847 \mathrm{e}-3$ \\
\hline 0.01 & 0.0316 & 1000 & $3.4160 \mathrm{e}-4$ & $9.7971 \mathrm{e}-2$ & $1.7896 \mathrm{e}-2$ & $9.3278 \mathrm{e}-3$ \\
\hline 0.05 & 0.1567 & 1000 & $7.2599 \mathrm{e}-3$ & 0.2467 & 0.1071 & $3.8046 \mathrm{e}-2$ \\
\hline 0.1 & 0.3308 & 1000 & $2.2546 \mathrm{e}-2$ & 0.4059 & 0.2067 & 0.1077 \\
\hline
\end{tabular}

Table 3: Numerical results for the finest grid $\ell=64$ and with different values of $\theta$.

In Figure 2 from left to right we show the computed numerical solution $q_{\ell}$ of the algorithm at the final iteration, and the differences $q_{\ell}-I_{1}^{h_{\ell}} q^{\dagger}, \mathcal{N}_{q_{\ell}}^{h_{\ell}} j_{\delta_{\ell}}-\mathcal{N}_{q^{\dagger}}^{h_{\ell}} j^{\dagger}$ and $\mathcal{D}_{q_{\ell}}^{h_{\ell}} g_{\delta_{\ell}}-\mathcal{D}_{q^{\dagger}}^{h_{\ell}} g^{\dagger}$ for $\ell=64$ and $\theta=0.005$, i.e., $\delta_{\ell}=0.0167$. Finally, Figure 3 performs the analog differences, but with $\theta=0.1$, i.e., $\delta_{\ell}=0.3308$.
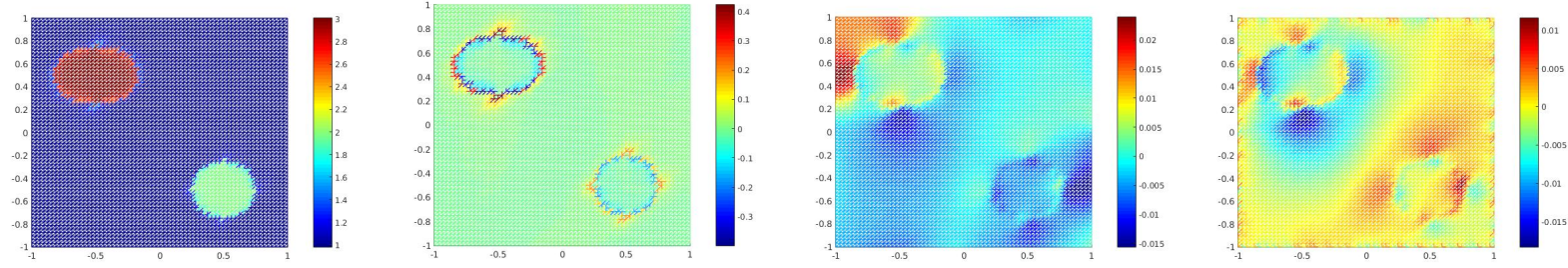

Figure 2: Computed numerical solution $q_{\ell}$ of the algorithm at the $991^{\text {th }}$ iteration, and the differences $q_{\ell}-I_{1}^{h_{\ell}} q^{\dagger}, \mathcal{N}_{q_{\ell}}^{h_{\ell}} j_{\delta_{\ell}}-\mathcal{N}_{q^{\dagger}}^{h_{\ell}} j^{\dagger}$ and $\mathcal{D}_{q_{\ell}}^{h_{\ell}} g_{\delta_{\ell}}-\mathcal{D}_{q^{\dagger}}^{h_{\ell}} g^{\dagger}$ for $\ell=64$ and $\theta=0.005$, i.e., $\delta_{\ell}=0.0167$. 

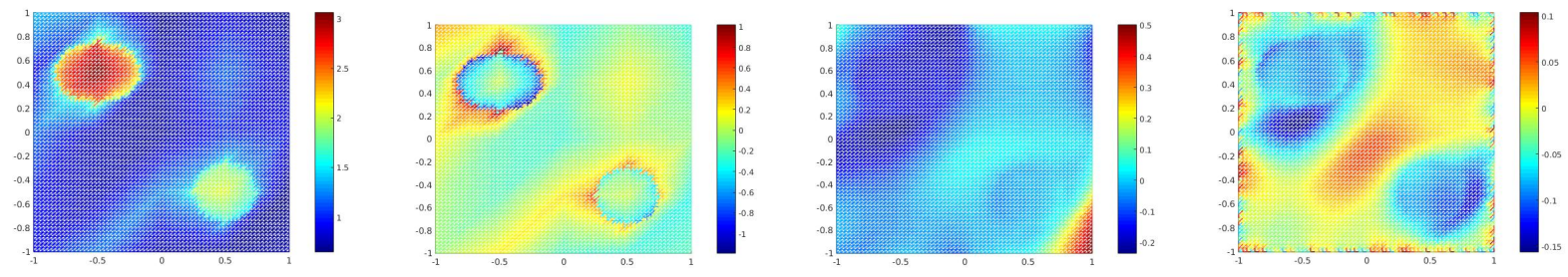

Figure 3: Computed numerical solution $q_{\ell}$ of the algorithm at the $1000^{\text {th }}$ iteration, and the differences $q_{\ell}-I_{1}^{h_{\ell}} q^{\dagger}, \mathcal{N}_{q_{\ell}}^{h_{\ell}} j_{\delta_{\ell}}-\mathcal{N}_{q^{\dagger}}^{h_{\ell}} j^{\dagger}$ and $\mathcal{D}_{q_{\ell}}^{h_{\ell}} g_{\delta_{\ell}}-\mathcal{D}_{q^{\dagger}}^{h_{\ell}} g^{\dagger}$ for $\ell=64$ and $\theta=0.1$, i.e., $\delta_{\ell}=0.3308$.

Example 5.3. In this example we assume that multiple measurements are available, say $\left(j_{\delta}^{i}, g_{\delta}^{i}\right)_{i=1, \ldots, I}$. Then, the cost functional $\Upsilon_{\rho, \delta}^{h}$ and the problem $\left(\mathcal{P}_{\rho, \delta}^{h}\right)$ can be rewritten as

$$
\min _{q \in \mathcal{Q}_{a d}^{h}} \bar{\Upsilon}_{\rho, \delta}^{h}(q):=\min _{q \in \mathcal{Q}_{a d}^{h}}(\underbrace{\frac{1}{I} \sum_{i=1}^{I} \int_{\Omega} q \nabla\left(\mathcal{N}_{q}^{h} j_{\delta}^{i}-\mathcal{D}_{q}^{h} g_{\delta}^{i}\right) \cdot \nabla\left(\mathcal{N}_{q}^{h} j_{\delta}^{i}-\mathcal{D}_{q}^{h} g_{\delta}^{i}\right)}_{:=\overline{\mathcal{J}}_{\delta}^{h}(q)}+\rho \int_{\Omega} \sqrt{|\nabla q|^{2}+\epsilon^{h}}), \quad\left(\overline{\mathcal{P}}_{\rho, \delta}^{h}\right)
$$

which also attains a solution $\bar{q}_{\rho, \delta}^{h}$. The Neumann boundary condition in the equation $(5.9)$ is chosen in the same form of (5.11), i.e.

$$
\begin{aligned}
j_{(A, B, C, D)}^{\dagger}= & A \cdot \chi_{(0,1] \times\{-1\}}-A \cdot \chi_{[-1,0] \times\{1\}}+B \cdot \chi_{(0,1] \times\{1\}}-B \cdot \chi_{[-1,0] \times\{-1\}} \\
& +C \cdot \chi_{\{-1\} \times(-1,0]}-C \cdot \chi_{\{1\} \times(0,1)}+D \cdot \chi_{\{1\} \times(-1,0]}-D \cdot \chi_{\{-1\} \times(0,1)},
\end{aligned}
$$

that depends on the constants $A, B, C$ and $D$. Let $g_{(A, B, C, D)}^{\dagger}:=\gamma \mathcal{N}_{q^{\dagger}} j_{(A, B, C, D)}^{\dagger}$ and assume that noisy observations are given by

$$
\left(j_{\delta_{\ell}}^{(A, B, C, D)}, g_{\delta_{\ell}}^{(A, B, C, D)}\right)=\left(j_{(A, B, C, D)}^{\dagger}+\theta \cdot R_{j_{(A, B, C, D)}^{\dagger}}, g_{(A, B, C, D)}^{\dagger}+\theta \cdot R_{g_{(A, B, C, D)}^{\dagger}}\right) \quad \text { with } \quad \theta>0,
$$

where $R_{j_{(A, B, C, D)}^{\dagger}}$ and $R_{g_{(A, B, C, D)}^{\dagger}}$ denote $\partial M^{h_{\ell}} \times 1$-matrices of random numbers on the interval $(-1,1)$.

With $\theta=0.1$ and $\ell=64$ the last line of Table 3 displays the numerical results for the case $(A, B, C, D)=$ $(1,2,3,4)$ and $I=1$, which is repeated in the first line of Table 4 for comparison.

We now fix $D=4$. Let $(A, B, C)$ be equal to all permutations of the set $\{1,2,3\}$. Then, the equations 5.13 -5.14 generate $I=6$ measurements. Similarly, let $(A, B, C, D)$ be all permutations of $\{1,2,3,4\}$ we get $I=16$ measurements. The numerical results for these two cases are presented in the two last lines of Table 4 respectively.

Finally, in Figure 4 from left to right we show the computed numerical solution $q_{\ell}$ of the algorithm at the final iteration for $\ell=64, \theta=0.1$, i.e., $\delta_{\ell}=0.3308$, and $I=1,6,16$, respectively.

\begin{tabular}{|c|l|l|l|l|l|}
\hline \multicolumn{7}{|c|}{ Numerical results for $\ell=64, \theta=0.1$} \\
\hline Number of observations $I$ & Iterate & Tolerance & $L_{q}^{2}$ & $L_{\mathcal{N}}^{2}$ & $L_{\mathcal{D}}^{2}$ \\
\hline 1 & 1000 & $2.2546 \mathrm{e}-2$ & 0.4059 & 0.2067 & 0.1077 \\
\hline 6 & 1000 & $8.5684 \mathrm{e}-3$ & 0.3159 & $7.4901 \mathrm{e}-2$ & $4.4704 \mathrm{e}-2$ \\
\hline 16 & 1000 & $4.0133 \mathrm{e}-3$ & 0.2547 & $5.6985 \mathrm{e}-2$ & $3.2211 \mathrm{e}-2$ \\
\hline
\end{tabular}

Table 4: Numerical results for $\ell=64, \theta=0.1$, i.e., $\delta_{\ell}=0.3308$, and with multiple measurements $I=1,6,16$. 

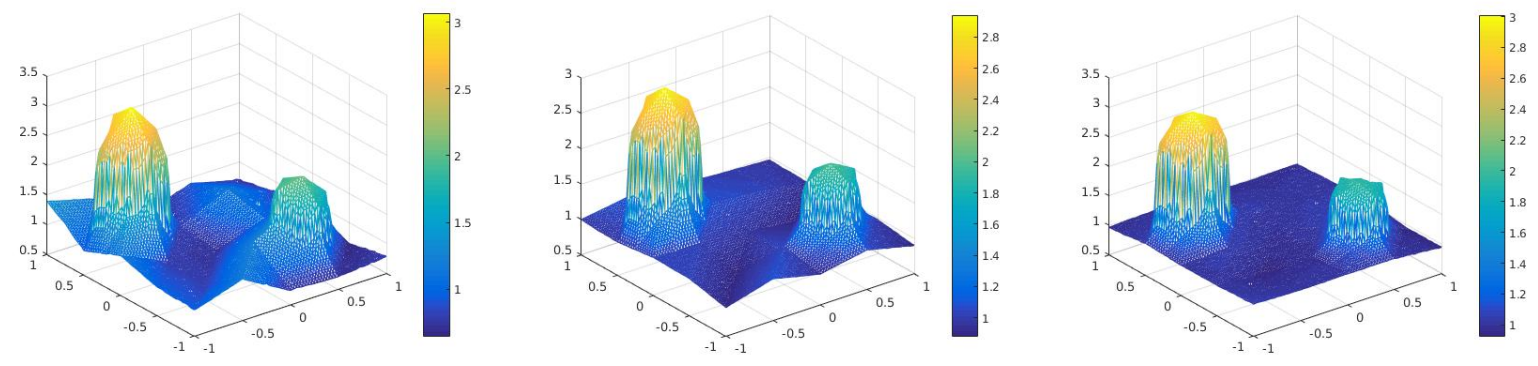

Figure 4: Computed numerical solution $q_{\ell}$ of the algorithm at the final iteration for $\ell=64, \theta=0.1$, i.e., $\delta_{\ell}=0.3308$, and with multiple measurements $I=1,6,16$, respectively.

We observe that the use of multiple measurements improves the solution to yield an acceptable result even in the presence of relatively large noise.

\section{Acknowledgments}

The authors M. Hinze, B. Kaltenbacher and T.N.T. Quyen would like to thank the referees and the editor for their valuable comments and suggestions which helped to improve our paper.

\section{References}

[1] R. A. Adams, Sobolev Spaces, New York San Francisco London: Academic Press, 1975.

[2] G. Alessandrini, V. Isakov, and J. Powell, Local uniqueness in the inverse conductivity problem with one measurement, Trans. Amer. Math. Soc. 347(1995), 3031-3041.

[3] K. Astala and L. Päivärinta, Calderón's inverse conductivity problem in the plane, Ann. Math. 163(2006), 265-299

[4] H. Attouch, G. Buttazzo and G. Michaille, Variational Analysis in Sobolev and BV Space, Philadelphia: SIAM, 2006.

[5] S. Bartels, R. H. Nochetto, and A. J. Salgado, Discrete TV flows without regularization, SIAM J. Numer. Anal. 52(2014), 363-385.

[6] C. Bernardi, Optimal finite element interpolation on curved domain, SIAM J. Numer. Anal. 26(1989), $1212-1240$.

[7] C. Bernardi and V. Girault, A local regularization operator for triangular and quadrilateral finite elements, SIAM J. Numer. Anal. 35(1998), 1893-1916.

[8] L. Blank and C. Rupprecht, An extension of the projected gradient method to a Banach space setting with application in structural topology optimization, Technical Report, ArXiv e-prints 2015 (http://arxiv.org/pdf/1503.03783v2.pdf).

[9] P. Bochev and R. B. Lehoucq, On the finite element solution of the pure Neumann problem, SIAM Rev. $47(2005), 50-66$.

[10] L. Borcea, Electrical impedance tomography, Inverse Problems 18(2002), 99-136.

[11] S. Brenner and R. Scott, The Mathematical Theory of Finite Element Methods, New York: Springer, 1994. 
[12] R. M. Brown and R. H. Torres, Uniqueness in the inverse conductivity problem for conductivities with $3 / 2$ derivatives in $L^{p}, p>2 n$, J. Fourier Anal. Appl. 9(2003), 563-574.

[13] R. M. Brown and G. A. Uhlmann, Uniqueness in the inverse conductivity problem for nonsmooth conductivities in two dimensions, Comm. Partial Differential Equations 22(1997), 1009-1027.

[14] M. Burger and S. Osher, A guide to the TV zoo, in: Level-Set and PDE-based Reconstruction Methods, M.Burger, S.Osher, eds.: Springer, 2013.

[15] A. P. Calderón, On an inverse boundary value problem, in Seminar on Numerical Analysis and its Applications to Continuum Physics (Rio de Janeiro, 1980), 65-73, Soc. Brasil. Mat., Rio de Janeiro, 1980.

[16] Z. Chen and J. Zou, An augmented Lagrangian method for identifying discontinuous parameters in elliptic systems, SIAM J. Control Optim. 37(1999), 892-910.

[17] M. Cheney, D. Isaacson, and J. C. Newell, Electrical impedance tomography, SIAM Rev. 41(1999), $85-101$.

[18] P. G. Ciarlet, Basis Error Estimates for Elliptic Problems, in Handbook of Numerical Analisis, Vol. II, P. G. Ciarlet and J. -L. Lions, eds., North-Holland Amsterdam: Elsevier, 1991.

[19] P. Clément, Approximation by finite element functions using local regularization, RAIRO Anal. Numér. 9(1975), 77-84.

[20] A. Dijkstra, B. Brown, N. Harris, D. Barber, and D. Endbrooke, Review: Clinical applications of electrical impedance tomography, J. Med. Eng. Technol. 17(1993), 89-98.

[21] D. C. Dobson, Recovery of blocky images in electrical impedance tomography, in Inverse Problems in Medical Imaging and Nondestructive Testing, H. W. Engl, A. K. Louis, and W. Rundell, eds.: Springer (1997), 43-64.

[22] H. W. Engl, M. Hanke, and A. Neubauer, Regularization of Inverse Problems, Dortdrecht: Kluwer, 1996.

[23] G. J. Fix, M. D. Gunzburger and J. S. Peterson, On finite element approximations of problems having inhomogeneous essential boundary conditions, Comp. ES Maths. with Appls. 9(1983), 687-700.

[24] E. Giusti, Minimal Surfaces and Functions of Bounded Variation, Boston: Birkhäuser, 1984.

[25] M. S. Gockenbach, Understanding and Implementing the Finite Element Method, Philadelphia: SIAM, 2006.

[26] P. Grisvard, Elliptic Problems in Nonsmooth Domains, Boston: Pitman, 1985.

[27] C. T. Kelley, Iterative Methods for Optimization, Philadelphia: SIAM, 1999.

[28] I. Knowles, A variational algorithm for electrical impedance tomography, Inverse Problems 14(1998), $1513-1525$.

[29] R. V. Kohn and A. McKenney, Numerical implementation of a variational method for electrical impedance tomography, Inverse Problems 6(1990) 389-414.

[30] R. V. Kohn and M. Vogelius, Determining Conductivity by Boundary Measurements, Comm. Pure Appl. Math. 37(1984), 289-298.

[31] R. V. Kohn and M. Vogelius, Determining conductivity by boundary measurements. II. Interior results, Comm. Pure Appl. Math. 38(1985), 643-667.

[32] R. V. Kohn and M. Vogelius, Relaxation of a variational method for impedance computed tomography, Comm. Pure Appl. Math. 40(1987), 745-777.

[33] J. L. Mueller and S. Siltanen, Linear and Nonlinear Inverse Problems with Practical Applications, Philadelphia: SIAM, 2012. 
[34] A. Nachman, Global uniqueness for a two-dimensional inverse boundary value problem, Ann. Math. 143(1996), 71-96.

[35] A. Neubauer and O. Scherzer, Finite-dimensional approximation of Tikhonov regularized solutions of nonlinear ill-posed problems, Numer. Funct. Anal. Optim. 11(1990), 85-99.

[36] K. Niinimäki, M. Lassas, K. Hämäläinen, A. Kallonen, V. Kolehmainen, E. Niemi and S. Siltanen, Multi-resolution parameter choice method for total variation regularized tomography, SIAM J. Imag. Sci. 9(2016), 938-974.

[37] L. Päivärinta, A. Panchenko, and G. Uhlmann, Complex geometric optics solutions for Lipschitz conductivities, Rev. Mat. Iberoamericana 19(2003), 57-72.

[38] C. Pechstein, Finite and Boundary Element Tearing and Interconnecting Solvers for Multiscale Problems, Heidelberg New York Dordrecht London: Springer, 2010.

[39] R. Scott and S. Y. Zhang, Finite element interpolation of nonsmooth function satisfying boundary conditions, Math. Comp. 54(1990), 483-493.

[40] E. Somersalo, M. Cheney, and D. Isaacson, Existence and uniqueness for electrode models for electric current computed tomography, SIAM J. Appl. Math. 52(1992), 1023-1040.

[41] J. Sylvester and G. Uhlmann, A global uniqueness theorem for an inverse boundary value problem, Ann. Math. 125(1987), 153-169.

[42] G. M. Troianiello, Elliptic differential equations and obstacle problems, New York: Plenum, 1987.

[43] G. Uhlmann, Electrical impedance tomography and Calderón's problem, Inverse Problems 25(2009), 123011 (39pp). 